\title{
Effects of Cavities and Protuberances on Transition over Hypersonic Vehicles
}

\author{
Chau-Lyan Chang, ${ }^{*}$ Meelan M. Choudhari, ${ }^{* *} \mathrm{Fei} \mathrm{Li}^{*}$ \\ NASA Langley Research Center, Hampton, VA 23681 \\ and Balaji Venkatachari** \\ University of Alabama at Birmingham, Birmingham, AL 35294
}

Surface protuberances and cavities on a hypersonic vehicle are known to cause several aerodynamic or aerothermodynamic issues. Most important of all, premature transition due to these surface irregularities can lead to a significant rise in surface heating. To help understand laminar-turbulent transition induced by protuberances or cavities on a Crew Exploration Vehicle (CEV) surface, high-fidelity numerical simulations are carried out for both types of trips on a CEV wind tunnel model. Due to the large bluntness, these surface irregularities reside in an accelerating subsonic boundary layer. For the Mach 6 wind tunnel conditions with a roughness Reynolds number $R e_{k k}$ of 800 , it was found that a protuberance with a height to boundary layer thickness ratio of 0.73 leads to strong wake instability and spontaneous vortex shedding, while a cavity with identical geometry only causes a rather weak flow unsteadiness. The same cavity with a larger Reynolds number also leads to similar spontaneous vortex shedding and wake instability. The wake development and the formation of hairpin vortices for both protuberance and cavity were found to be qualitatively similar to that observed for an isolated hemisphere submerged in a subsonic, low speed flat-plate boundary layer. However, the shed vortices and their accompanying instability waves were found to be slightly stabilized downstream by the accelerating boundary layer along the CEV surface. Despite this stabilizing influence, it was found that the wake instability spreads substantially in both wall-normal and azimuthal directions as the flow is evolving towards a transitional state. Similarities and differences between the wake instability behind a protuberance and a cavity are investigated. Computations for the Mach 6 boundary layer over a slender cylindrical roughness element with a height to the boundary layer thickness of about 1.1 also shows spontaneous vortex shedding and strong wake instability. Comparisons of detailed flow structures associated with protuberances at subsonic and supersonic edge Mach numbers indicate distinctively different instability mechanisms.

\section{Nomenclature}

$A \quad=$ area of the space-time element interface

$D \quad=$ depth of the cavity, or diameter of the cylindrical roughness element

$e \quad=\quad$ total energy, defined in the second section

$f \quad=$ frequency of instability oscillations

$F, G, H \quad=$ inviscid flux vectors

$F_{v}, G_{v}, H_{v}=$ viscous flux vectors

$\vec{h} \quad=$ flux in the joint space-time domain

$k=$ cylinder height for both protuberance and cavity

$L \quad=$ length of the cavity

$M \quad=$ freestream Mach number

$p \quad=$ pressure

$p_{e} \quad=$ boundary layer edge pressure

$p_{o} \quad=$ stagnation pressure

$Q \quad=$ dependent solution vector, defined in the second section

$R \quad=$ universal gas constant

Re $\quad=$ Reynolds number per unit length

\footnotetext{
* Aerospace Technologist, MS 128, Computational AeroSciences Branch, Senior Member, AIAA

${ }^{* *}$ Aerospace Technologist, MS 128, Computational AeroSciences Branch, Associate Fellow, AIAA

*** Post-Doctoral Research Fellow, Department of Mechanical Engineering, Member, AIAA
} 


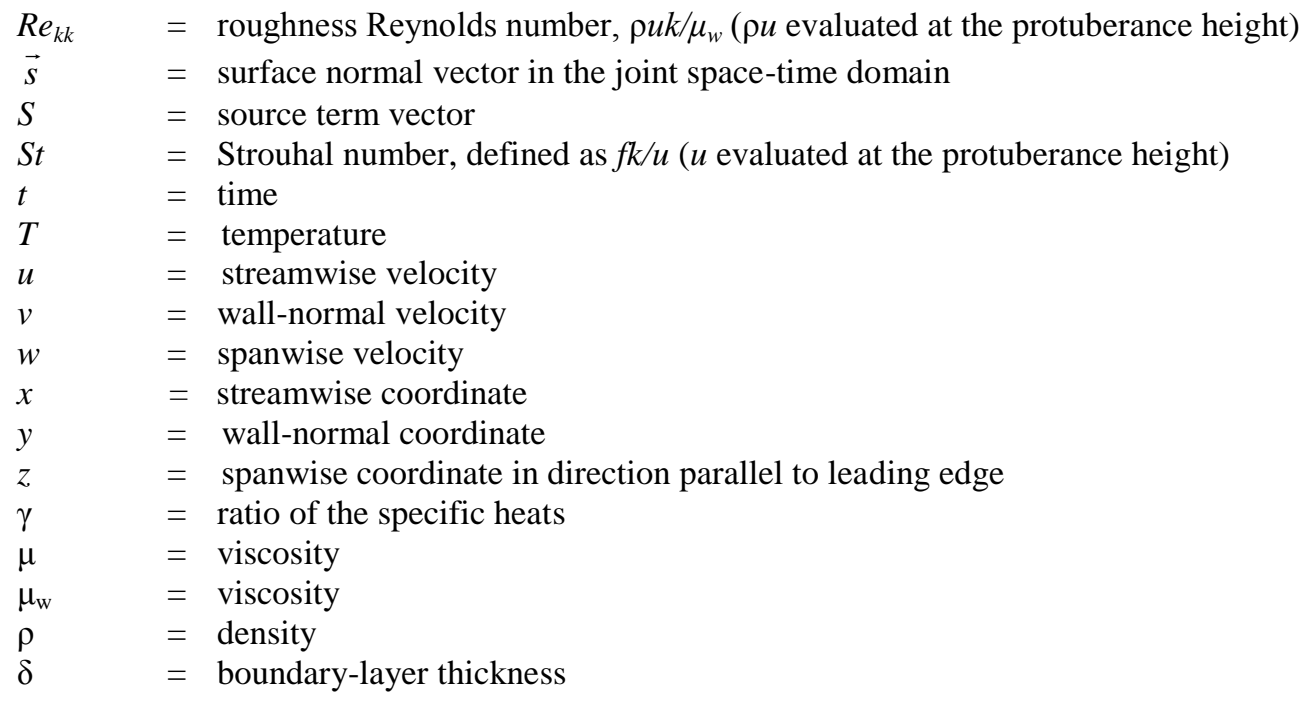

\section{Introduction}

$\mathrm{H}^{\mathrm{s}}$ ypersonic wake flow behind roughness elements has been actively investigated during the past few years due to its importance in many hypersonic applications. ${ }^{1-5}$ It is well-known that the presence of protuberances or cavities in hypersonic boundary layers can lead to premature laminar turbulent transition in the wake region. Early transition to turbulence causes a rapid rise in surface heating, which in turn may impact the aerodynamic and aerothermodynamic performance of the vehicle. Unfortunately, such irregularities are common to many hypersonic vehicles due to gap fillers and other manufacturing defects in the thermal protection system (TPS). As a result, effects of cavities and protuberances on surface heating must be accounted for in the TPS design.

Numerical computations of the transitional flowfield behind such surface irregularities in a hypersonic boundary layer are non-trivial because of the intricate flow physics involved. Conventional design of the TPS system thus relied on empirical formulations derived from wind-tunnel tests and sparse flight data. To enhance the predictive capabilities and further the understanding of the flow physics, a combination of experimental measurements and higher fidelity numerical computations of viscous flow over large surface irregularities are needed. At hypersonic speeds, a roughness element with a height smaller than the boundary layer thickness serves as a mean flow modifier which can alter the stability characteristics of the boundary layer. It has been shown, on the other hand, that the wake becomes strongly unsteady when the roughness height is substantially larger than the boundary layer thickness. ${ }^{3}$ When the roughness height exceeds the boundary-layer thickness, instability waves begin to develop in the wake region, causing the wake streak and horse-shoe vortices to oscillate downstream. At even larger heights, the instability waves may eventually lead to vortex breakdown. Strong vortex shedding behind an isolated roughness element at relatively small Reynolds numbers is evident in some of the recent computational studies ${ }^{5-6}$. Interestingly, it was identified in Ref. 5 that the origin of strong wake instability appears to be a likely absolute instability taking place in the front-side separation region of the roughness element. Recent wind tunnel experiments also confirm the existence of instability waves upstream of a slender cylindrical roughness element attached to the Mach 6 tunnel wall. $^{7}$

In contrast to protuberances, effects of cavities on hypersonic boundary layers are relatively less understood. Wind tunnel experiments have been performed for transition correlations of cavities on Shuttle Orbiter transition. ${ }^{8-9}$ However, only transition fronts discerned from surface thermography for selected cavity geometries are available from these experiments. To enhance the predictive capability, detailed computational studies similar to the aforementioned roughness investigations are warranted. For applications of slender body vehicles, the boundary layer edge Mach number is mostly in the hypersonic regime. On the other hand, for large blunt bodies such as a reentry capsule or the crew exploration vehicles (CEV), surface irregularities in the form of cavities or protuberances may exist in the subsonic flow regime as well. Ablation-induced irregularities could also potentially cause early transition near the stagnation region. In these flows, however, the mass ejection from the ablating wall could also play a significant role on premature transition in addition to the geometrical changes. Therefore, the Mach 
number of interest in the investigations of cavities and protuberances should range from subsonic to hypersonic speeds.

When a large surface roughness is submerged in a hypersonic stream, complex flow physics such as shock-shock interactions, shock-boundary layer interactions, flow separation, unsteady shear layers and wakes may develop around and downstream of the roughness element site. To cope with the geometries of arbitrary roughness shapes, the time-accurate tetrahedral mesh conservation element, solution element (CESE) method ${ }^{10-13}$ is adopted for unsteady Navier-Stokes calculations using the NASA in-house CESE Navier-Stokes solver, $e z 4 d^{12-13}$. Unlike conventional upwind methods, the CESE method is a genuine multi-dimensional scheme formulated by enforcing flux conservation in the discretized space and time domain. Integration of the conservation laws is carried out on staggered discretized volumes called conservation elements (CE). Consequently, no interface reconstruction or dimensional-splitting is needed. Flux conservation in space and time offers distinctly better numerical properties, both in terms of boundary condition treatment and temporal accuracy.

The main objective of this research is to investigate effects of cavities and protuberances on boundary-layer transition over hypersonic vehicles. Both subsonic and supersonic flow regimes are studied for surface irregularities pertinent to both slender as well as large blunt hypersonic vehicles. In particular, effects of protuberance and cavity on a CEV model in the subsonic flow regime will be investigated in detail. Computational results provide flowfield details and instability characteristics that can help understand flow physics and in particular, interpret the data measured from the experiments. The present computational investigation will be focused on the exploration of flow physics. Cavity wake development in a subsonic boundary layer and its effectiveness in tripping the hypersonic boundary layers will be studied via time-accurate Navier-Stokes computations. As an on-going effort from our previous studies, effects of large, slender roughness elements will also be investigated for the Purdue Mach 6 cylindrical roughness experiments. ${ }^{7}$ Computational results in this paper are aimed at furthering the knowledge base for hypersonic vehicle transition and enhancing predictive capabilities for complex flow physics in hypersonic flows via improvements of numerical methods and computational software development. In the next section, the numerical method used is discussed, followed by a description of the CEV configuration studied. Results and discussion and summary are presented in Sections IV and V, respectively.

\section{Numerical Method}

Three-dimensional compressible Navier-Stokes equations in vector form can be written as

$$
\frac{\partial Q}{\partial t}+\frac{\partial F}{\partial x}+\frac{\partial G}{\partial y}+\frac{\partial H}{\partial z}=S+\frac{\partial F_{v}}{\partial x}+\frac{\partial G_{v}}{\partial y}+\frac{\partial H_{v}}{\partial z}
$$

where the dependent variable vector is defined as $Q=(\rho, \rho u, \rho v, \rho w, e)$ and $x, y, z$, and $t$ represent spatial coordinates and time, respectively. Flow variables $\rho, u, v, w$, and $e$ represent density, three velocity components, and total energy $\left(e=p /(\gamma-1)+\rho\left(u^{2}+v^{2}+w^{2}\right) / 2\right.$, where $p$ denotes the pressure $)$, respectively. Definitions of the inviscid flux vectors $F, G, H$ and viscous flux vectors $F_{v}, G_{v}, H_{v}$ can be found in standard text books and will not be included here. The source vector $S$ contains all external forcing or other energy-related source terms. To close the system, the perfect gas relation, $p=\rho R T$, with $T$ representing the temperature, is used in conjunction with eq. (1).

The space-time CESE method is formulated in the strong form of the flux equations. The space-time flux $\vec{h}$ is defined as:

$$
\vec{h}=\left(F-F_{v}, G-G_{v}, H-H_{v}, Q\right)
$$

By using Gauss' divergence theorem in the space-time domain, Eq. 1 is rewritten in the following integral form

$$
\oint_{\sigma(V)} \vec{h} \cdot d \vec{s}=\int_{\mathrm{v}} S d V
$$


where the space-time flux vector is integrated over the surface $\sigma$ of an arbitrary space-time volume $V$. The surface normal vector is defined by $\vec{s}=\vec{n} d A$, where $d A$ is the area increment on $\sigma$, and $\vec{n}$ is the outward unit normal vector. Equation 3 is quite general, and in fact, any conservation law with or without a source vector can be cast in this form. Thus, the numerical algorithm devised for Eq. 3 can be easily extended to other physical problems.

The discretized domain is divided into two different kinds of discretized sub-volumes. Numerical solution of the dependent variables within a 3D solution element is assumed to satisfy the following first order equation:

$$
Q(x, y, t)=Q_{0}+Q_{t}\left(t-t_{0}\right)+Q_{x}\left(x-x_{0}\right)+Q_{y}\left(y-y_{0}\right)+Q_{z}\left(z-z_{0}\right)
$$

where $Q_{0}$ is the solution vector at the solution point $O$. The vectors $Q_{t}, Q_{x}, Q_{y}$, and $Q_{z}$ are analogous to the derivatives of the dependent variables. In the CESE method, the solution element coincides with the discretized cell. It is either triangles or quadrilaterals in two dimensions, and tetrahedrons or hexahedrons in three dimensions. The first-order Taylor series expansion used here ensures uniformly second-order accuracy in both space and time. In contrast to the conventional unstructured mesh method where flux integration is done on these elements directly, integration of the conservation laws in the CESE method is carried out on a series of integration volumes called conservation elements. The solution elements, quadrilaterals in $2 \mathrm{D}$ and pyramids or prisms in $3 \mathrm{D}$, are staggered to the solution elements in the generalized space-time domain. Conventional schemes, including the discontinuous Galerkin method, utilize 1D approximate Riemann solutions to uniquely determine the flux vectors at all the cell interfaces. Dimensional splitting with certain flavor of locally one-dimensional assumptions must be employed for multi-dimensional flows. The cell interfaces of the staggered conservation elements in the CESE method, on the other hand, are located entirely within the solution elements where dependent variables are uniquely defined. Therefore, numerical integration can be carried out in the smooth regions without any Riemann solvers to handle jump solutions between cells. More details of the numerical formulations can be found in the literature. ${ }^{10-13}$

Formulations for general nonlinear equations in the CESE method are constructed based on a non-dissipative core scheme that satisfies the space-time inversion invariant property. Three distinct features make the CESE method superior to conventional schemes in numerical accuracy. Firstly, flux conservation is enforced over the space and time domain synergistically. Such treatment intrinsically improves temporal accuracy and the extra flux propagation in time makes the implementation of non-reflecting boundary conditions much easier. Secondly, numerical formulation of the CESE method is designed to handle multi-dimensional unstructured meshes without introducing any ad-hoc Riemann approximations and special monotonicity-preserving techniques. Flux integration over the staggered conservation elements ensures that numerical fluxes are always uniquely determined at all cell interfaces. As a result, all numerical issues related to the interface flux treatment do not exist in the CESE method. Thirdly, numerical dissipation control is achieved in reference to the non-dissipative core scheme. High solution accuracy can thus be obtained by reducing numerical dissipation as desired.

Numerical computations in this research have been carried out by using the $e z 4 d$ code, which is developed in the $\mathrm{C}++$ language entirely by following modern software engineering procedures. Object-oriented and generic programming using templates have been employed extensively in the software framework for $e z 4 d$. Sound software architecture ensures easy maintenance and agile future development. Both message passing interface (MPI) and multi-thread capabilities are integrated in the software framework to take advantages of multi-core, distributed memory cluster environment. Currently, the $e z 4 d$ code can handle Euler or laminar Navier-Stokes equations for triangular, quadrilateral, tetrahedral, or hexahedral unstructured meshes. The baseline algorithm in the $e z 4 d$ code has been modified slightly for viscous flow computations. ${ }^{13}$ The original strong flux conservation formulation in conjunction with a modified edge-base derivatives approach is used to effectively compute viscous flow with highly stretched unstructured meshes inside the boundary layer. Solution accuracy has been carefully validated for timeaccurate inviscid and viscous flows.

One of the advantages of the CESE method is the ease of implementing subsonic boundary conditions. Due to the space-time conservation properties, the CESE method allows simple implementation of subsonic boundaries without incurring strong boundary reflections. At these boundaries, the prescribed dependent variables are imposed at the ghost cell adjacent to the boundaries and their spatial derivatives are either set to zero or extrapolated from inside the domain. Figure 1 shows an example of computing subsonic viscous flow over a 2D airfoil by using both full and truncated domains. The truncated domain has close proximity of the downstream boundaries to the trailing edge of 
the airfoil. However, only very minor reflection near the truncated boundaries can be observed. The converged steady state solutions from two grids agree quite well for the most part of the domain despite the poor locations of the downstream boundaries in the artificially truncated domain. No additional buffered domain is necessary as the extent of boundary influence is only limited to the elements near the truncated boundaries. This nice numerical property allows the use of prescribed subsonic inflow and outflow boundary conditions for the CEV configurations to be discussed below. Boundary reflection of sensitive quantities such as acoustic waves also takes place at subsonic boundaries. According to previous investigations ${ }^{13}$ in the extreme case, acoustic wave reflection only occurs within a few wave lengths near the boundaries. For the protuberance and roughness simulations of interest in this study, the subsonic inflow and outflow boundaries are sufficiently far away from the perturbation source and thus minor boundary reflection will not be an issue for all time-accurate calculations.

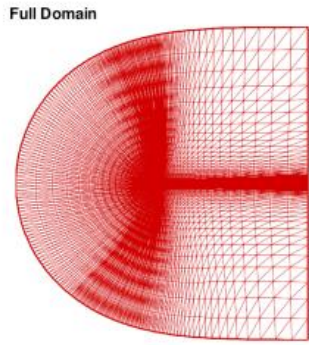

(a)

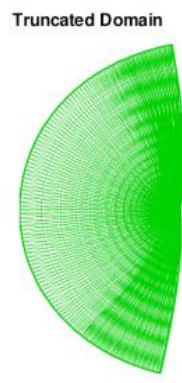

(b)
Transonic (Mach 0.85) flow over an RAE airfoil

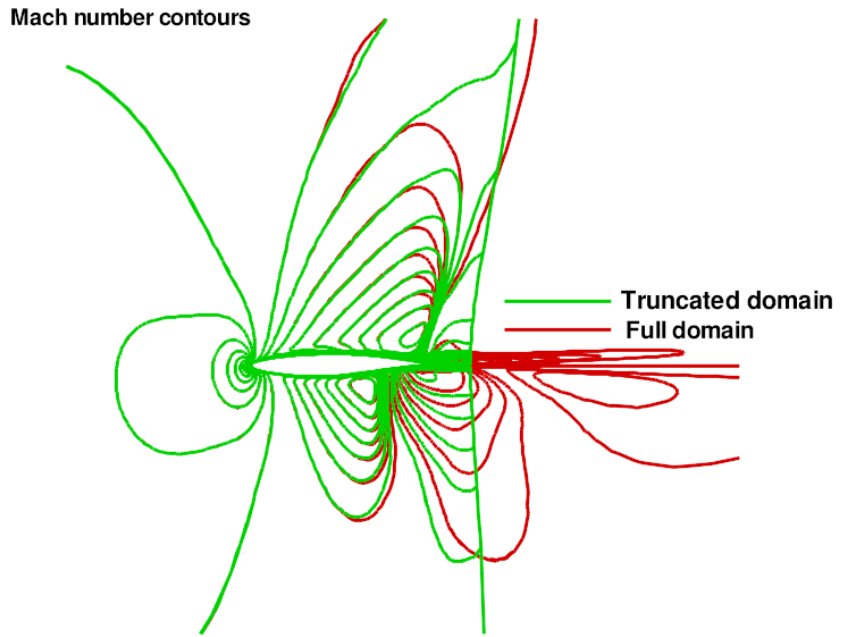

(c)

Figure 1. Mach 0.85 flow over an RAE airfoil with a $2.72^{\circ}$ angle of attack computed with triangular elements (a) complete domain (b) truncated domain (c) comparison of computed Mach number contours

To more efficiently compute the CEV configurations with a protuberance or a cavity within the subsonic boundary layer located behind the bow shock, a truncated domain that encloses the shock and the trip with both subsonic inflow and outflow boundaries is used for direct numerical simulations. Subsonic inflow and outflow profiles computed with a larger domain that captures the entire flowfield around the CEV model (from centerline to supersonic outflow beyond the shoulder) are imposed at the subsonic boundaries of the truncated domain. It will be shown later that such approach allows the inviscid solution of the truncated domain to match the desired inviscid solution of the entire CEV model while the viscous solution within or in the vicinity of the boundary layer is modified by the presence of the protuberance or cavity. The use of such "domain within the domain" computations significantly reduces the overall grid counts for the direct numerical simulations.

\section{CEV Configurations and Boundary Conditions}

Effects of protuberances on slender vehicles can be effectively studied by computing a hypersonic flat-plate boundary layer over a roughness element, as was done in previous investigations. ${ }^{3,5}$ In this paper, we focus on the effects of protuberances or cavities on large blunt bodies, in particular, NASA's Project Orion crew entry vehicle configuration. The CEV model as shown in Fig. 2 has been experimentally investigated in the NASA Langley Research Center, 20-inch Mach 6 tunnel. ${ }^{17}$ The pizza-box shape protuberance array was used to trip the laminar boundary layers at several wind tunnel conditions. Measured surface heating data along the symmetry axis was used to determine the corresponding transition locations. Both $24^{\circ}$ and $28^{\circ}$ angle of attack cases were measured. To mimic the realistic flow conditions, the highest Reynolds number case with a free stream Mach number of 6 , free- 
stream temperature of $60 \mathrm{~K}$, and a free-stream unit Reynolds number of $2.62 \times 10^{7} / \mathrm{m}$ (this corresponds to $8 \times 10^{6} / \mathrm{ft}$ ) was chosen for this study. The wall temperature is assumed to be $300 \mathrm{~K}$. Without loss of detailed transition physics along the leeward symmetry axis where most experimental data was measured, zero angle of attack is assumed in order to simplify the base flow computations and inflow/outflow profile interpolations. Steady state solution as shown in Fig. 3(a) for the above axisymmetric configuration was computed by the VULCAN code. ${ }^{18}$ As a preliminary attempt to understand transition physics, the trip array is simplified to a single cylindrical roughness element which inscribes one pizza box. The trip location is set to give the same $k / \delta$ value as the trip E at the same wind-tunnel condition in the experiment. ${ }^{17}$ Based upon the computed axisymmetric meanflow, the trip is located at around $45 \%$ of the CEV radius from the center where the boundary-layer edge Mach number is about 0.3 , as opposed to an estimated edge Mach number of about 0.8 for the trip E location in Ref. 17. The roughness height is estimated to be $0.1466 \mathrm{~mm}$ and the cylinder diameter is $1.128 \mathrm{~mm}$, using the same $k / D$ ratio of 0.13 as in the experiment. The resulting roughness Reynolds number $R e_{k k}$ is around 440 and the roughness height to boundary layer thickness ratio $(k / \delta)$ is about 0.54 . Nonetheless, most of the results presented in this paper were computed with a slightly larger height with an $\operatorname{Re}_{k k}$ of 800 for reasons to be discussed later. Details of this other configuration will be described in the next section.

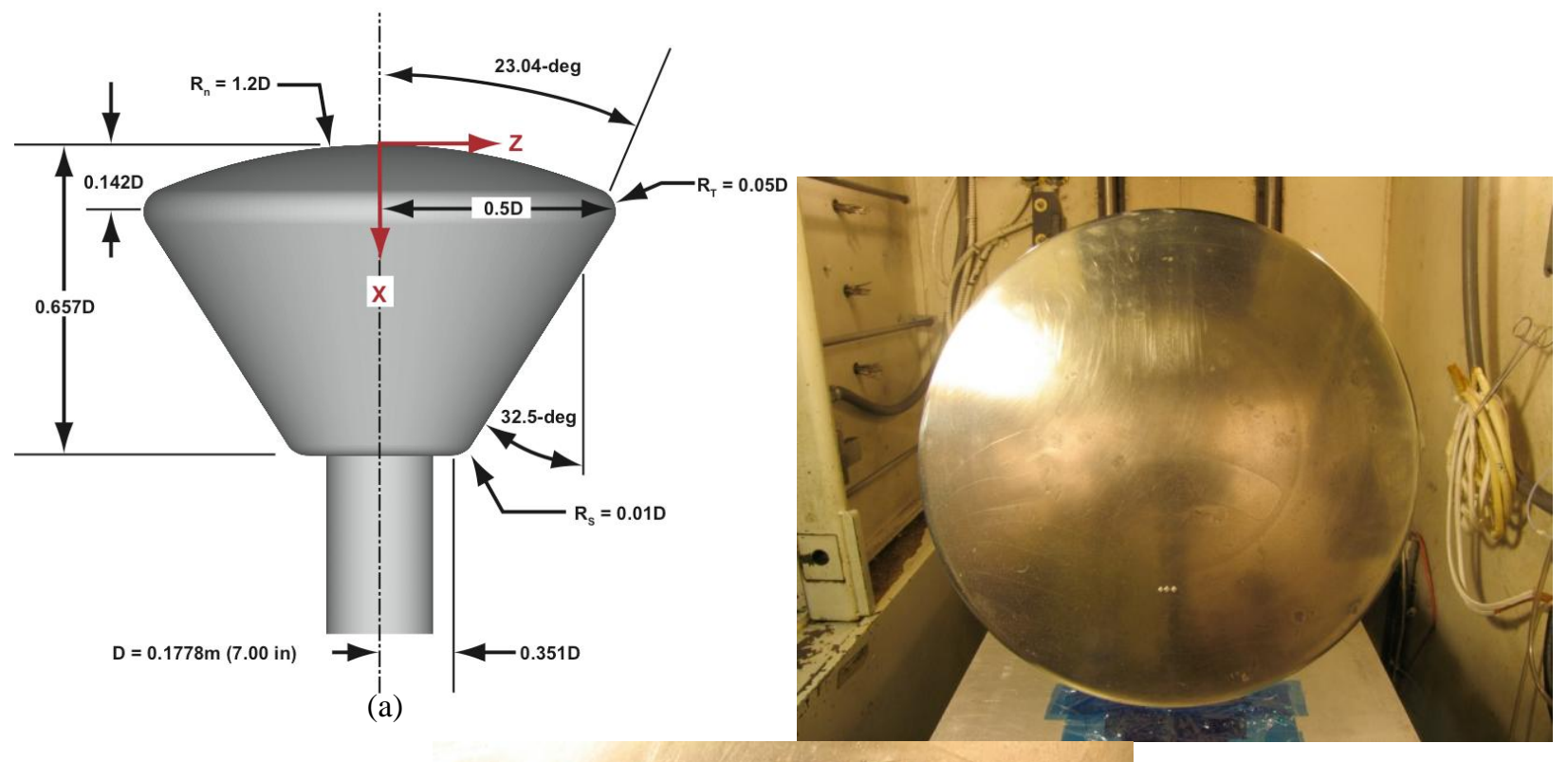

(b)

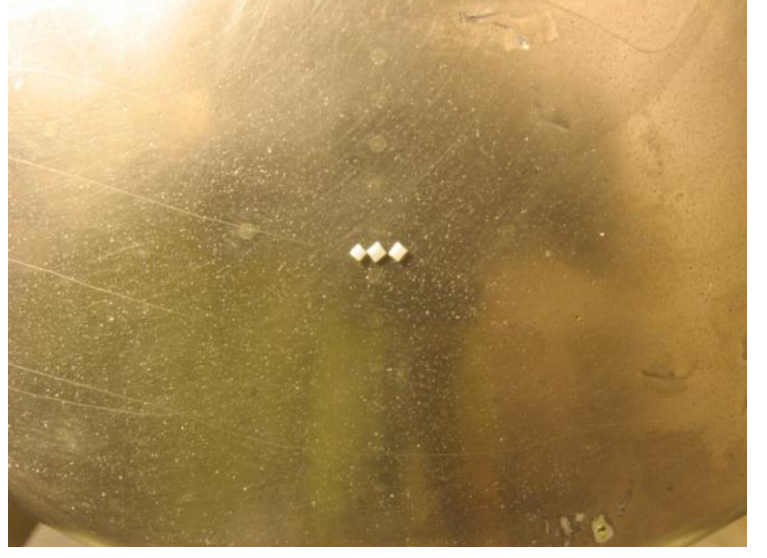

(c)

Figure 2. Project Orion crew entry vehicle wind tunnel model as investigated in Amar et al ${ }^{17}$ (figures taken from the same reference) (a) model dimension (b) heatshield (c) pizza-box shape trip-array

The numerical simulation for the trip (protuberance or cavity) is done with a truncated computational domain as shown in Fig. 3(a). This domain extends significantly far away from the wall to include the bow shock. For the 
CEV configuration, the sonic line is located very close to the edge of the model. Both the inflow (bottom) and outflow (top) boundaries are thus in the subsonic region. Computations could be done by moving the outflow boundary to the supersonic region near the edge of the model. However, doing so would significantly increase the computational cost. The numerical boundary conditions imposed are as those depicted in Fig. 3(b). At the subsonic inflow and outflow boundaries, the computed steady-state axisymmetric solutions from VULCAN are imposed and derivatives are set to zero. The supersonic free-stream conditions and no-slip conditions are imposed at the left and right boundaries, respectively. Symmetry boundary conditions are imposed at both azimuthal boundaries and only half of the protuberance or cavity is computed. Note that the results presented in the next section are shown for the mirrored full protuberance or cavity domain. Non-reflecting boundary conditions were also attempted for the subsonic outflow boundaries with other boundaries unchanged. It was found that the bow shock did not stay in position as the time was advanced. Imposing the subsonic outflow conditions fixed the correct pressure distributions, which in conjunction with the supersonic free-stream conditions and the body geometry gives the desired truncated domain flow solution as if it is a portion of the full CEV configuration. Note that both the inflow and outflow profiles could also be computed using the $e z 4 d$ code. The intent here is demonstrate how two different flow solvers could work together for a high-fidelity simulation by taking advantage of the rapid convergence to steady state by an implicit flow solver.

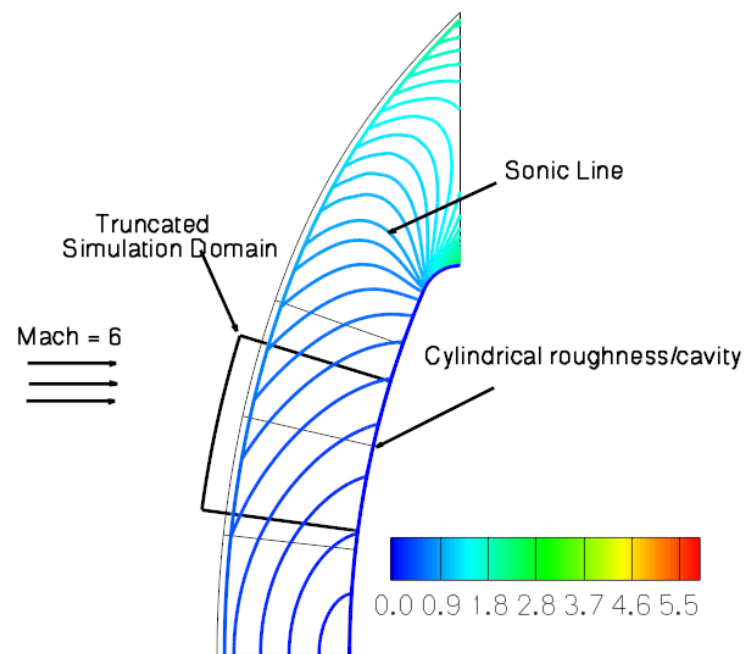

(a)

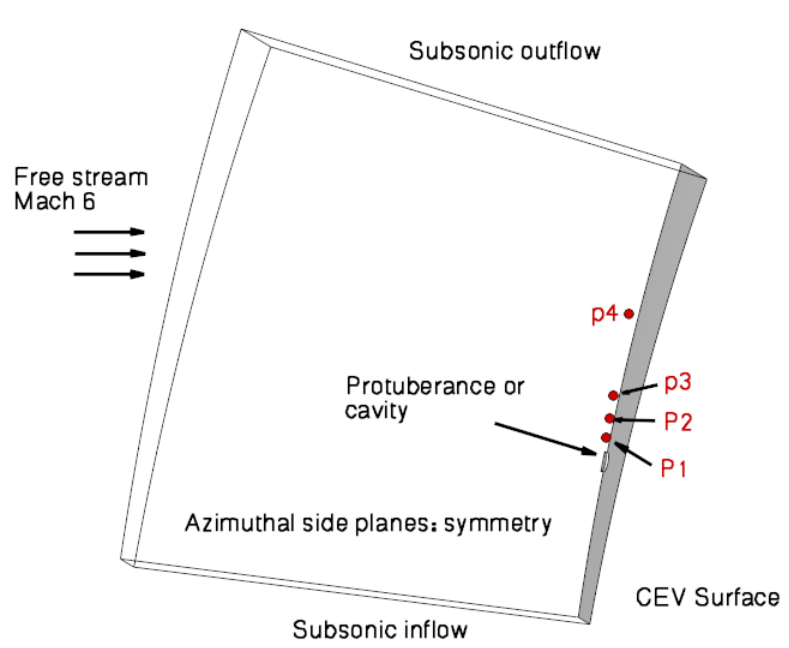

(b)

Figure 3. Mach 6 flow over the CEV model with zero angle of attack (axisymmetric) (a) computed Mach number contours in the full and truncated domains (b) enlarged view of the truncated computational domain and boundaries

Figure 4 shows comparison of the computed smaller domain, axisymmetric $e z 4 d$ solution with the complete domain, VULCAN solution. In addition to the flow variations due to the roughness, only minor boundary effects are evident near the subsonic inflow and outflow boundaries. The bow shock stays in the correct position after a long time integration. Throughout the course of this study, the bow shock position is monitored to ensure the correct bluntbody solution over the entire CEV configuration has been computed with the presence of either a protuberance or cavity. 


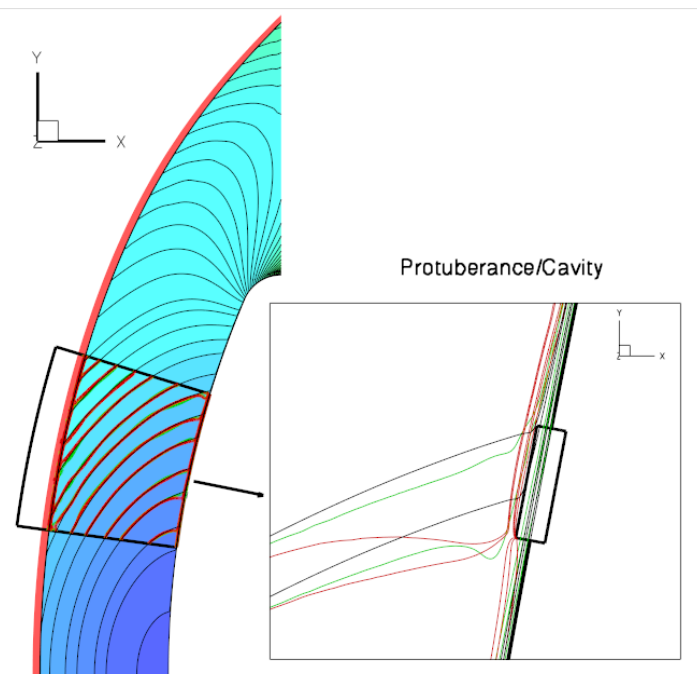

(a)

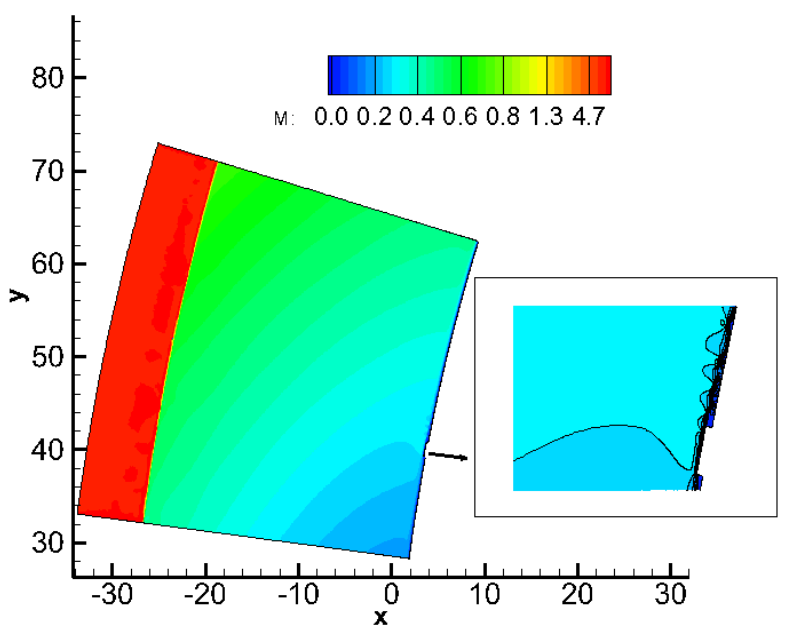

(b)

Figure 4. Comparison of computed Mach number contours between the baseline VULCAN solution and the present solution with an axisymmetric protuberance or cavity (a) superposition of all three solutions (b) Mach number contours of the protuberance case, showing effectiveness of the subsonic boundary conditions

\section{Results and Discussion}

After the verification of the boundary conditions, a series of two-dimensional simulations using triangular mesh were performed for an axisymmetric protuberance/cavity strip for parametric studies. Such 2D configurations may not be relevant for realistic configurations. However, they provide a quick means to ensure proper boundary conditions are imposed and provide a guideline of required grid resolution for full 3D tetrahedral meshes. Results from 2D and preliminary 3D numerical computations using relatively coarse grid with the conditions described in Section III for $R_{k k}=440$ indicate that the wake flow of both the protuberance and the cavity is only marginally unstable even though experimental results for the three pizza-box trip indicate transition right behind the roughness elements for the trip E location ${ }^{17}$ with a higher boundary layer edge Mach number around 0.8. NASA Ames Research Center's ballistic range experimental results for a hemisphere ${ }^{19}$ suggest that for an isolated disk-like trip, the critical $R e_{k k}$ value to trigger transition is around 800 for air. Measurements were done with a free-stream Mach number is 12 and boundary layer edge Mach number around the trip location varies from 0.25 to 0.75 . They also found that distributed roughness elements are more effective trips than the isolated ones. Using these conclusions as guidelines, the $R e_{k k}$ value for both the protuberance and the cavity is increased to 800 by changing the height $k$ to $0.1978 \mathrm{~mm}$ and maintaining the same $k / D$ ratio of 0.13 . The roughness height to boundary layer thickness ratio $(k / \delta)$ is also increased to about 0.73 based on the VULCAN solution shown above. All the results presented hereafter were computed using the new trip dimensions.

Axisymmetric Protuberance/Cavity

Subsonic boundary layer over a two-dimensional protuberance or cavity has been an active subject of research for decades. It has been shown in previous investigations ${ }^{3}$ that a rectangular roughness element submerged in a subsonic flat-plate boundary layer causes spontaneous vortex shedding downstream, similar to what happens in the wake of a backward facing step. ${ }^{20}$ Flow over a cavity has been investigated actively for the acoustic resonance mechanisms. For a proper cavity length to height ratio, the cavity also leads to spontaneous vortex shedding downstream. ${ }^{14,25-26}$ Both experimental and computational investigations have been conducted extensively in order to understand the flow physics. Although not shown here, a sequence of computations for subsonic flat-plate boundary layers over a rectangular cavity were parametrically studied to establish grid requirements and assess solution accuracy. The knowledge base established was then used as a guideline for the computations presented herein. The 2D simulations were done using a triangular mesh with about 330,000 and 570,000 elements for protuberance and cavity, respectively. The height and width of the axisymmetric protuberance and cavity are taken as the height and diameter of the corresponding 3D geometry noted above. Note that for an axisymmetric configuration, a protuberance or cavity with a rectangular cross section appears as a roughness ring or groove on the CEV surface. 


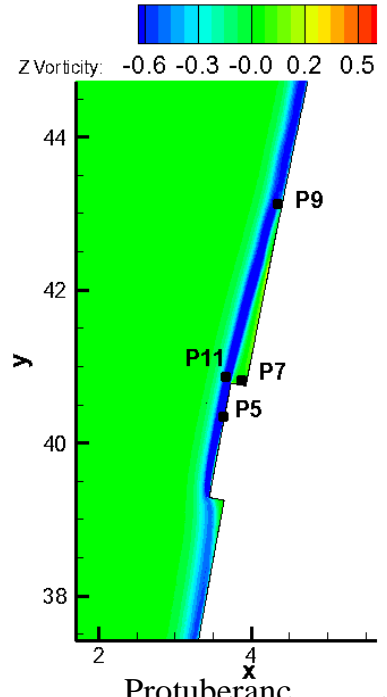

Protuberanc

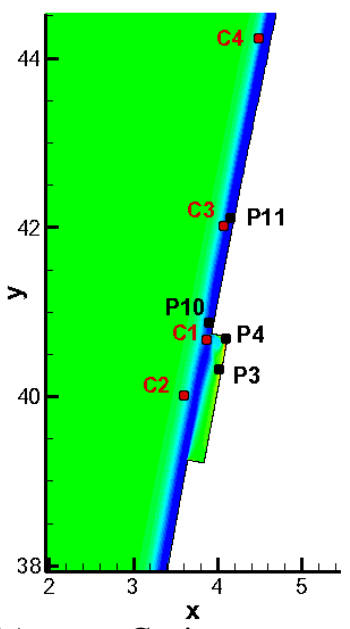

(a)

Cavity

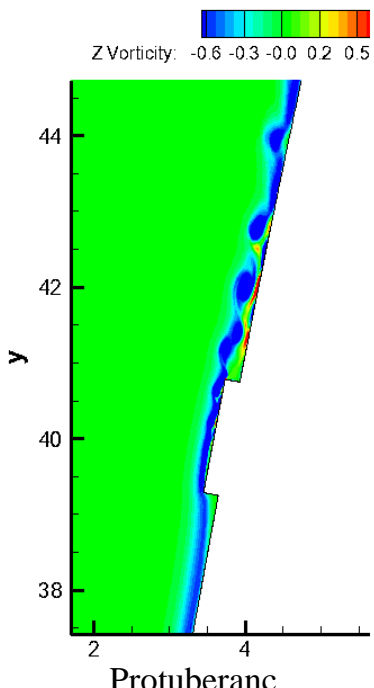

Protuberanc

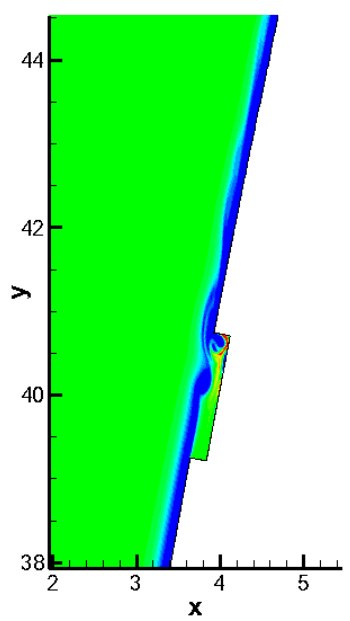

(b)

Cavity

Figure 5. Instantaneous vorticity contours for axisymmetric (2D) roughness strip (protuberance or cavity): (a) experimental condition at $R e_{k k}=800$ (b) at $\operatorname{Re}=2.62 \times 10^{8} / \mathrm{m}$

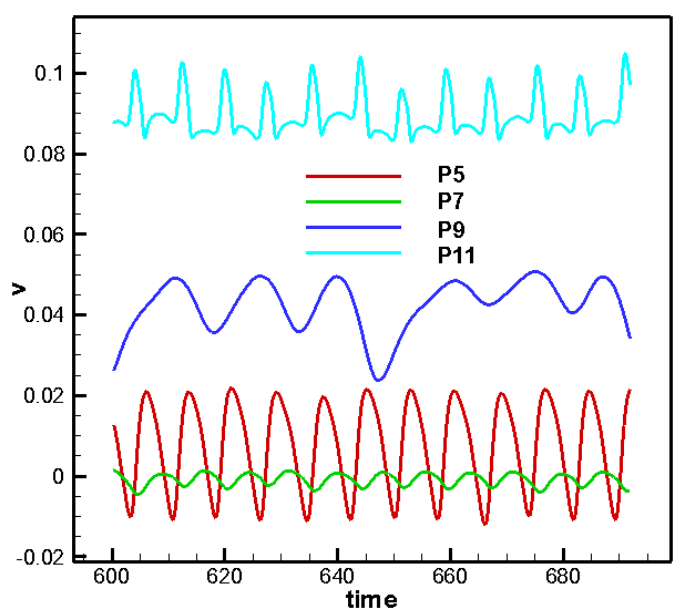

(a)

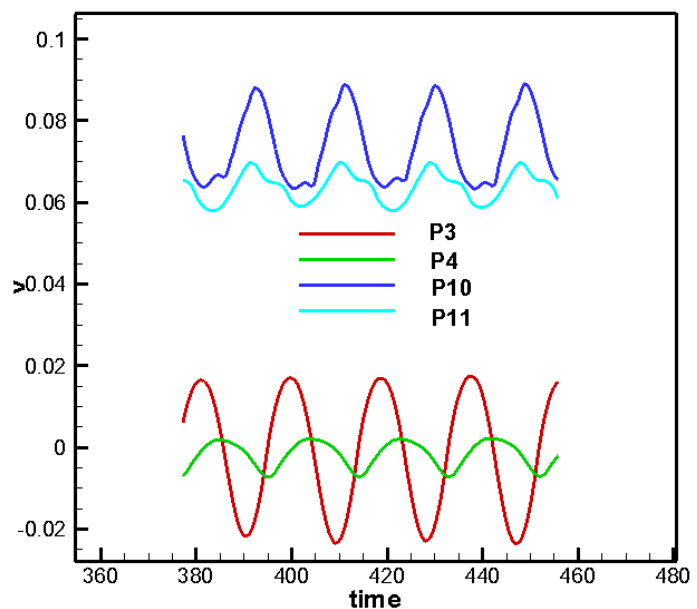

(b)

Figure 6. Time history of the v-velocity (roughly align with the CEV surface) at representative probe locations (shown in Fig. 5(a)) for the vortex shedding results at $\mathrm{Re}=\mathbf{2 . 6 2 \times 1 0 ^ { 8 }} / \mathrm{m}$ : (a) protuberance (b) cavity

Unlike the flat-plate boundary layers, the roughness strips on a CEV surface are situated in a strong favorable pressure gradient region. Subsonic flow behind the bow shock of the CEV capsule accelerates to supersonic speed as it approaches the shoulder. The flow acceleration might have stabilizing influence on the wake instability. Figure 5(a) shows the computed vorticity contours at the experimental flow conditions for both protuberance and cavity. The wake of the flow is only slightly unstable, indicated by weak flapping of the downstream shear layer. No vortex shedding is observed for either protuberance or cavity. Figure 5(b) shows the results obtained by increasing the unit Reynolds number by 10 times but maintain the same boundary layer thickness at the inflow boundary. Such combination is equivalent to a protuberance or cavity located at further downstream of the CEV surface at the higher Reynolds number. The $k / \delta$ ratios for the higher Reynolds number cases are estimated to be around 0.9. Spontaneous vortex shedding is evident for both configurations. The time history of the v-velocity (which roughly aligns with the CEV surface) shown in Fig. 6 exhibits quasi steady-state patterns at a constant frequency for several probes located in the vicinity of the protuberance or cavity as shown in Fig. 5(a). Further downstream (probe P9 in Fig. 5(a)), a lower frequency is observed for protuberance. The dominant frequencies are $122 \mathrm{kHz}$ and $47 \mathrm{kHz}$, for protuberance and cavity, respectively. The predicted Strouhal number for the protuberance 
is thus 0.205 (using $f k / u$, with $u$ evaluated at the roughness height), in line with Ref. 22-24. For the cavity case, the predicted Strouhal number is 0.59 (using edge velocity), which is within the range of correlated data in Ref.14. This value is lower than the first Rossiter's mode ${ }^{14}$ of 0.86 . According to the DNS data base for subsonic flat-plate cavities ${ }^{14}$ the $R e_{k k}=800$ case would have a weak instability without vortex shedding at a free-stream Mach number of 0.6 and a length to depth ratio around 8. The simulation results for 2D roughness strips shown in Fig. 5(a) appear to be in line with the DNS results.

\section{D Cylindrical Protuberance/Cavity}

Based upon the axisymmetric simulations results, a 3D tetrahedral mesh was generated for a cylindrical protuberance and cavity with the dimensions mentioned above. The domain size is as shown in Fig. 3(b). As noted above, only half of the protuberance or cavity is computed. Such half domain analysis is appropriate for instability wave simulations but somewhat questionable for full turbulent flows. The wall-normal direction extends about $15 \%$ above the bow shock and the azimuthal domain is about 3 cylinder diameters from the symmetry plane. The streamwise domain extends from about 10 diameters upstream to about 20 diameters downstream of the trip. The total mesh count is about 62 million and 68 million tetrahedrons for the protuberance and the cavity, respectively. To ensure solution quality, isotropic tetrahedrons are generated throughout the computational domain instead of those sliced from high-aspect ratio hexahedrons. Figure 7 shows the protuberance mesh along with the computed surface limiting streamline pattern and vorticity contours in the symmetry plane. Flow instability and vortex shedding are evident for the protuberance. Similar computations for the cavity yield mild instability in the wake region. The shear layer around the cavity became unsteady but no vortex shedding was observed.

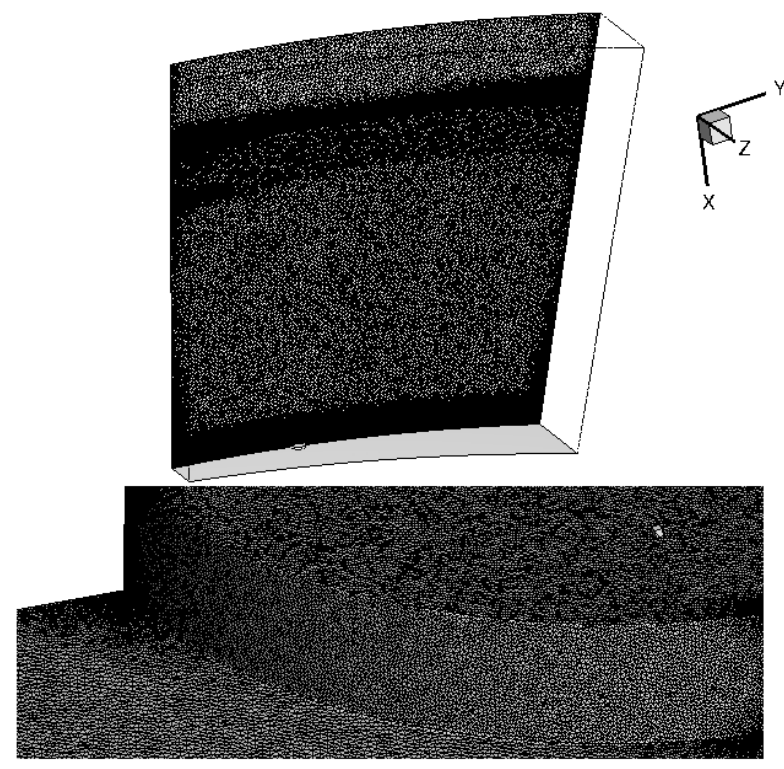

(a)

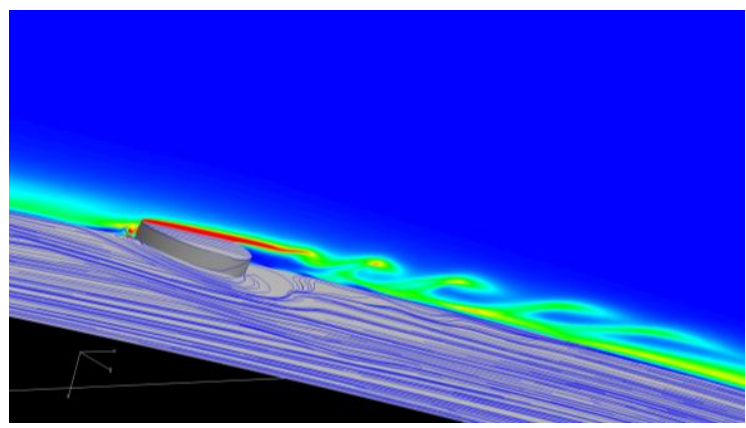

(b)

Figure 7. Tetrahedral mesh and computational results for the $3 D$ protuberance at $R e_{k k}=800$ (a) 62 million tetrahedral mesh (b) computed limiting streamline on the surface and vorticity contours

The results obtained from the above mesh also suggest that the domain of influence of the trip (both upstream and downstream) is smaller than what was estimated during the grid generation process. Based on these computational results, a smaller domain, more refined mesh which extends about 5 diameters upstream, 10 diameters downstream, and about 2.5 diameters from the symmetry plane in the spanwise direction was generated. More tetrahedrons were placed in the wake region to more accurately resolve the instability wave structure (see the improved cavity mesh in Fig. 8). Relatively uniform size tetrahedrons with a protuberance height to the mesh spacing ratio of about 100 have been placed in the wake region from the wall to about twice the boundary-layer thickness. The total mesh count is about 62 and 66 million for protuberance and cavity, respectively. Almost identical mesh counts with a smaller domain and better mesh distribution allows the flow physics to be better resolved. Simulation results for the $\operatorname{Re}_{k k}=$ 800 conditions using the refined mesh yield similar results, i.e., spontaneous vortex shedding is only evident for the protuberance. The cavity only causes mild instability, signified by small-amplitude flapping of the shear layer on 
top of the cavity and unsteadiness in the near wake region. This weak instability of the cavity resembles that observed for the axisymmetric cavity strips shown above in Fig. 5(a) at the same Reynolds number. Threedimensionality is apparently an important ingredient for the instability waves of the protuberance at this flow condition. A finite size cylindrical protuberance is much more unstable than an equivalent axisymmetric roughness strip at the same flow conditions. The computational results shown here also confirm that at $\operatorname{Re}_{k k}=800$ and a $k / \delta$ ratio of 0.73, the cylindrical protuberance on the CEV surface at a free-stream Mach number of 6 would lead to flow instability and spontaneous vortex shedding but an equivalent cavity does not.

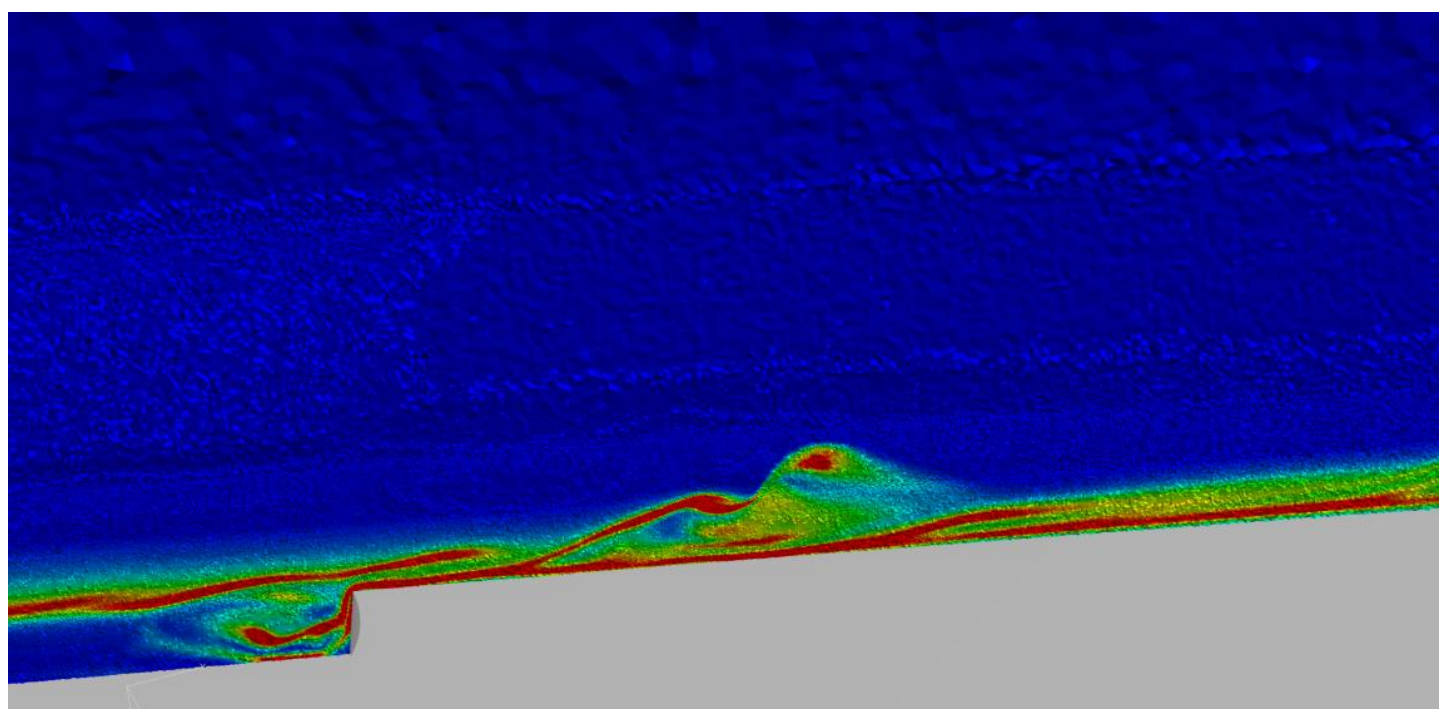

Figure 8. Improved tetrahedral mesh for the cavity, showing crinkles on top of the computed vorticity contours at a unit Reynolds number of $2.62 \times 10^{8} / \mathrm{m}$ near the symmetry plane.

To monitor the wake flow development, time histories of several probes located on top of the trip and near wake as shown in Figs. 3(b) and 5(a) for the protuberance and cavity, respectively, are recorded during the simulations. These probe points are all located near the symmetry plane. Figure 9 shows the time history of the v-velocity at several probe locations for the protuberance and cavity. Both show flow unsteadiness. The protuberance case has reached an almost quasi-steady state. The time traces for the less unstable cavity case have not yet reached the largetime asymptotic behavior as in the 2D cases (see Fig. 6). Nevertheless, flow structures do not vary too much for both cases. There appears to be a dominant frequency of about $115 \mathrm{kHz}$ (and its harmonics) for the protuberance. The estimated disturbance frequency has peaks around 42 and $70 \mathrm{kHz}$ for the cavity case. Additional computations at a higher Reynolds number indicate similar multiple peaks behavior after a nearly asymptotic state has been reached. For low speed flow over a hemisphere, it has been found both computationally ${ }^{21-22}$ and experimentally $y^{23-24}$ that there exists a dominant frequency associated with the shedding of the hairpin vortex. The single tone instability of the protuberance case seems to be similar to their results. However, the present protuberance is a shallow cylindrical roughness element with sharp edges which could potentially introduce some differences. Whether or not the broader band of disturbance frequencies for the cavity case is mainly a Reynolds number or cavity geometry effect remains to be investigated by further calculations.

The computed instantaneous surface limiting streamlines and surface heating patterns are depicted in Fig. 10. Comparison with the supersonic flow over a protuberance ${ }^{3,5}$ indicates that the upstream separation region for the present subsonic case is much smaller. It is interesting to note that similar horseshoe vortices wrapping around the cylindrical protuberance are also observed in the present subsonic case, except that they are closer to the centerline. Surface heating rate is substantially higher along these vortices than near the center line (Fig. 10(b)). Near the end, the heating rate appears to decay. In contrast, no horseshoe vortices are observed and the surface heating rate is higher only in the immediate wake of the cavity, as expected from its weak instability. The heating rate distribution shown here is only a snapshot, time-averaged solutions could be different. Figure 11 compares the vorticity magnitude contours at the symmetry plane for the protuberance and cavity. The shear layer remains attached to the protuberance surface. About one diameter downstream, it begins to shed vortices. These vortices travel downstream and form a consecutive lambda shape high shear layer at about one to two cylinder heights. For the 
cavity, a small-scale vortex is formed inside the cavity. However, the boundary layer remains attached to the surface in the wake region. The disturbances originating from the cavity only cause very small amplitude oscillation downstream.

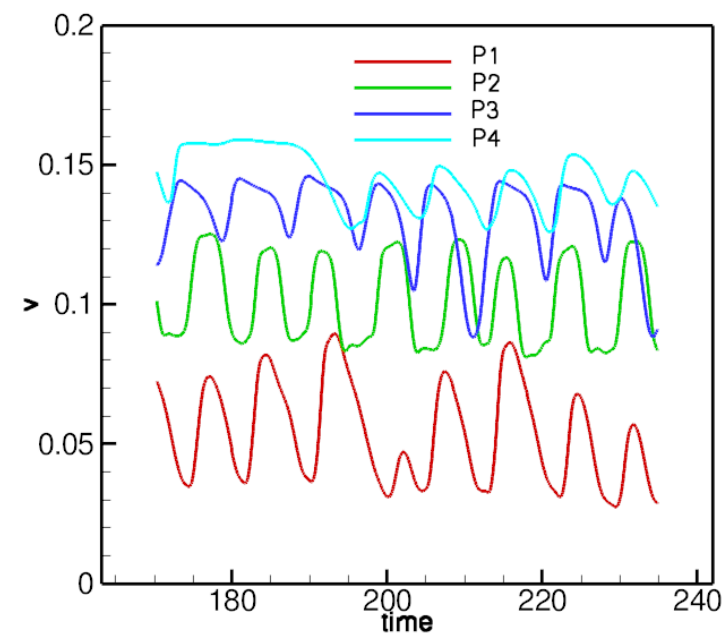

(a)

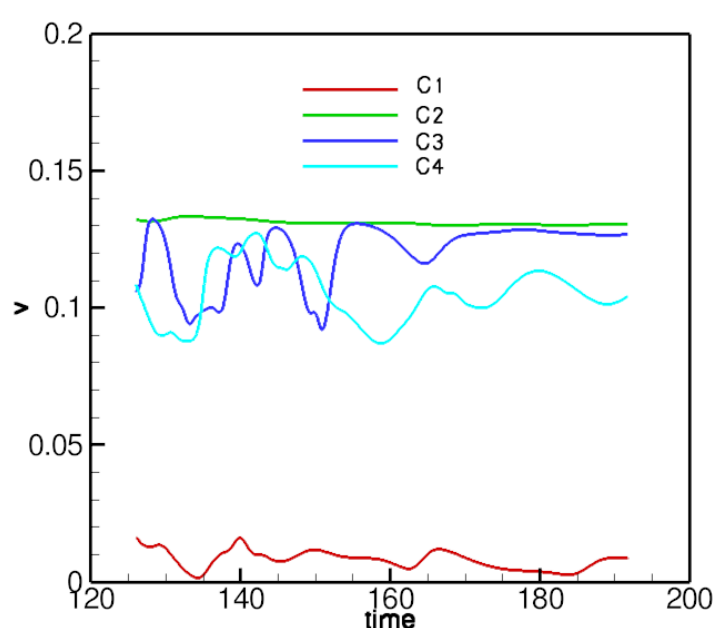

(b)

Figure 9. Time history of v-velocity at four probes located near the symmetry plane: (a) protuberance (probe locations shown in Fig. 3(b)) (b) cavity (probe locations shown in Fig. 5(a))

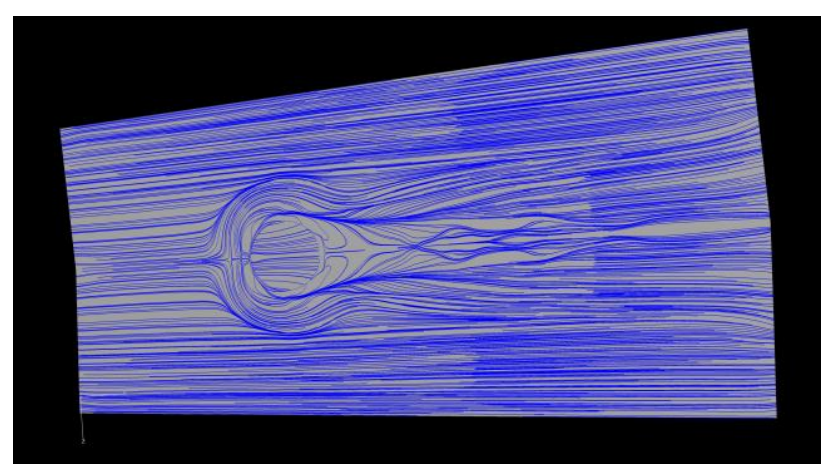

Protuberance

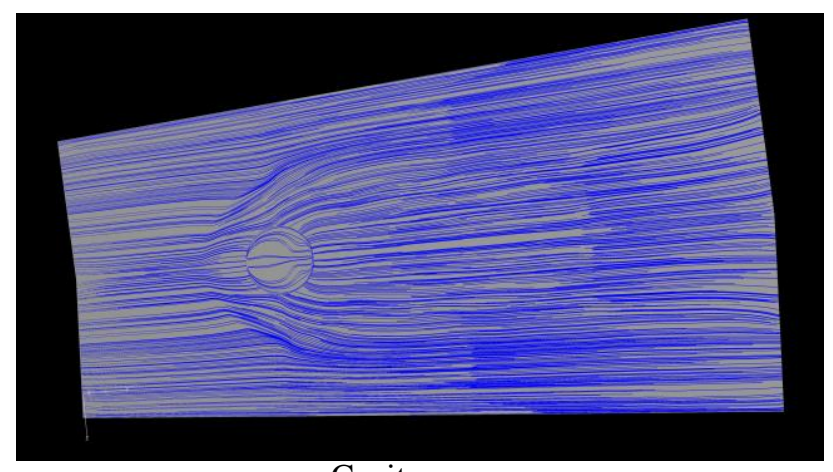

Cavity

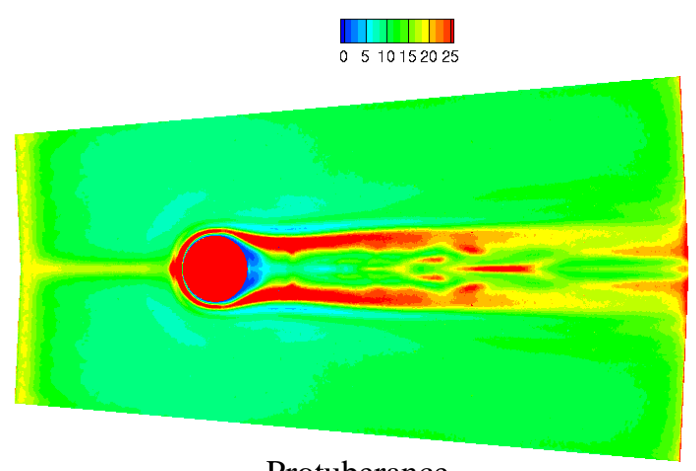

Protuberance

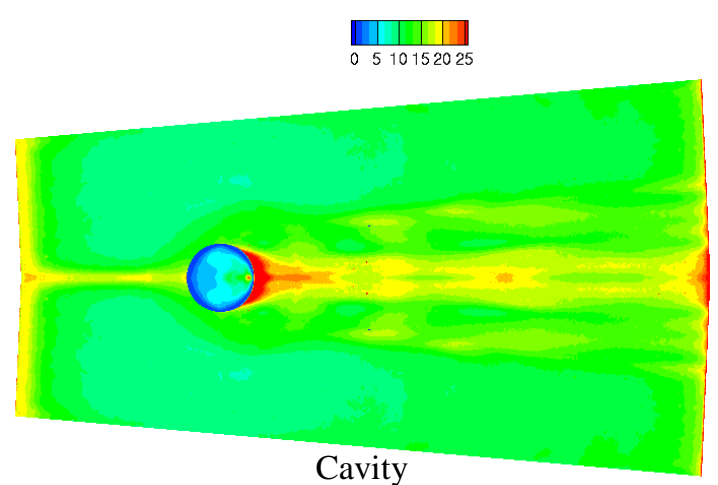

(b)

Figure 10. Comparison of surface limiting streamlines and surface heating patterns for protuberance and cavity, top plots are for protuberance and bottom ones for cavity: (a) surface limiting streamline pattern (b) normalized wall temperature gradient (proportional to surface heating)

Vorticity magnitude and $\mathrm{Q}$ value (the second invariant of the velocity gradient tensor ${ }^{27}$ ) isosurfaces are plotted in Fig. 12. These vortex patterns give good indications on how the unstable vortices are developing and propagating 
downstream of the trip. In addition to the horseshoe vortices, the vortex structure wraps around the cylindrical protuberance and sheds hairpin vortices at certain distinct frequency downstream. These hairpin vortices travel downstream and appear to decrease its intensity as they approach the end of the domain. The appearance of the hairpin vortices at a distinct frequency as shown in the snapshot is very similar to that observed in the low-speed experiments ${ }^{21,23}$ for a hemisphere protuberance submerged in a flat-plate boundary layer. In contrast, only small size vortex ripples are observed for the cavity case shown in Fig. 12(b). No eddy motion exists in the vicinity of the cavity as shown in the Q-value plot. For both protuberance and cavity, the wake region (domain of influence downstream of the trip) extends to about three diameters wide along the azimuthal direction near the end of the domain. Flow characteristics for the protuberance case can be further observed in the instantaneous streamline patterns and the vorticity contours on a constant wall-distance plane shown in Fig. 13. The streamlines show spiral motions both along the horseshoe vortices and the near centerline vortex. As seen in Fig. 13(b), consecutive lambda shape vortices are traveling downstream near the centerline. The intensity begins to decrease at about half of the distance between the protuberance and the end of the domain. Similar decaying trends are also observed in Figs. 10(b), 11(a), and 12(a).

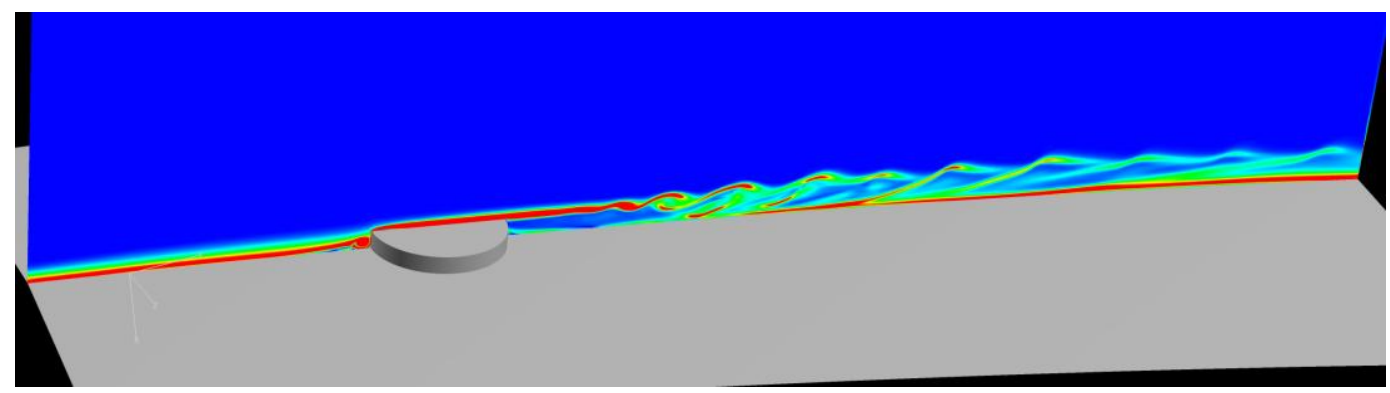

(a)

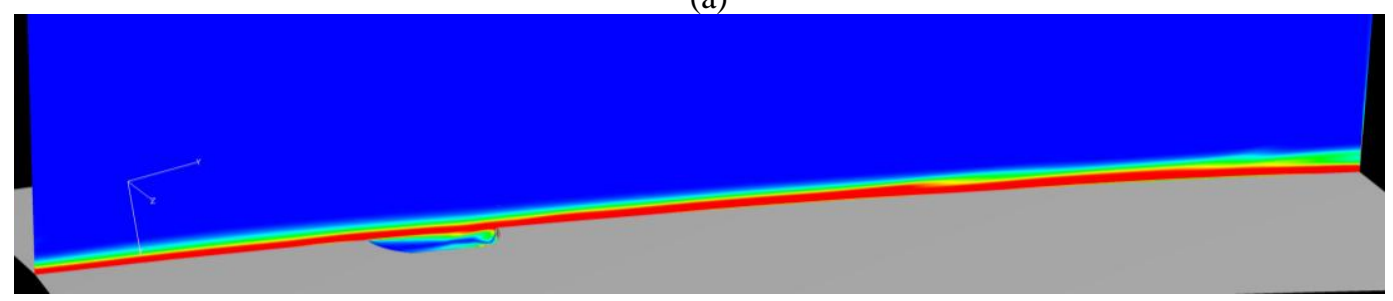

(b)

Figure 11. Comparison of instantaneous vorticity contours at the symmetry plane for protuberance and cavity (a) protuberance (b) cavity

To further investigate this decaying trend, the computed rms of the v-velocity is plotted against the y-coordinate (see Fig. 4(b) for the coordinate system used) in Fig. 14(a) for all the mesh points in the symmetry plane. The flow is quiet upstream of the protuberance. Disturbances rapidly grow to the first peak at about $1.5 \%$ rms amplitude (normalized by the free-stream velocity of $931 \mathrm{~m} / \mathrm{sec}$, the value increases to $10.6 \%$ if normalized by the boundary layer edge velocity at Mach 0.3 ) right after the protuberance. Two additional peaks at $2.1 \%$ and $1.8 \%$ take place further downstream. Disturbances appear to decay after reaching the peak values. However, maximum disturbances maintain at around $1.2 \%$ near the end of the domain. The overall rms amplitude drops from a maximum of $2.1 \%$ to $1.2 \%$ in the symmetry plane. Although not shown here, disturbances continue to grow in the entire wake region at some other azimuthal planes (but with a smaller peak values around $1.5 \%$ ). The v-velocity rms distribution shown in Fig. 14(b) reveals that the large disturbances, upon generation, spreads towards the wall rapidly and maintains energetic from the wall to about twice the protuberance height. Moreover, the disturbance field shown in Fig. 14(c) for several downstream constant y-coordinate planes (which make an about $83^{\circ}$ angle with respective to the CEV surface) continues to develop and spread in the azimuthal direction until the end of the domain. The multiple-vortex structure near the end indicates that the flow remains highly unstable near the wall. The peak perturbation location moves closer to the wall as the flow approaches the end of the domain. The slight decay in the rms amplitude seems to confirm earlier observations drawn from instantaneous solutions. One likely cause of this stabilizing effect is the favorable pressure gradient of the subsonic boundary layer as the flow accelerates to a supersonic speed along the CEV surface. The rms distribution also indicates that, in contrast to the supersonic cylindrical roughness element simulated previously ${ }^{5}$, the small separation region upstream of the cylinder does not play a role in the instability 
wave development. Strong instability manifested by vortex shedding takes place downstream of the roughness element in a subsonic boundary layer. This fundamental difference leads to completely different protuberance wake instability mechanisms between large blunt bodies and slender vehicles in the supersonic flow regime.
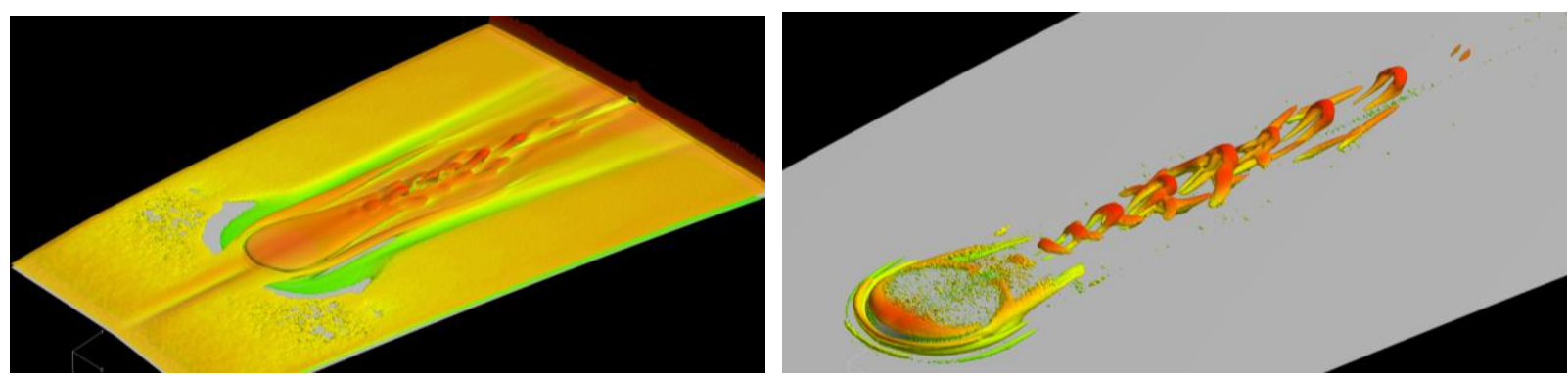

(a)
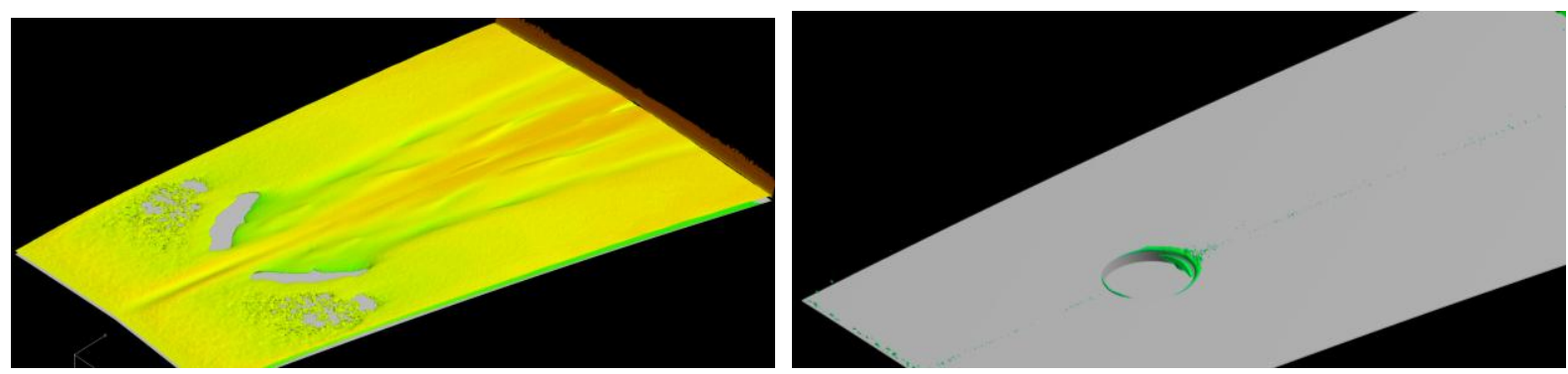

(b)

Figure 12. Comparison of instantaneous vorticity magnitude (left, non-dimensional value of 0.5 ) and $Q$ value (right, second invariant of velocity gradient tensor, non-dimensional value of 0.01 ) isosurfaces (a) protuberance (b) cavity

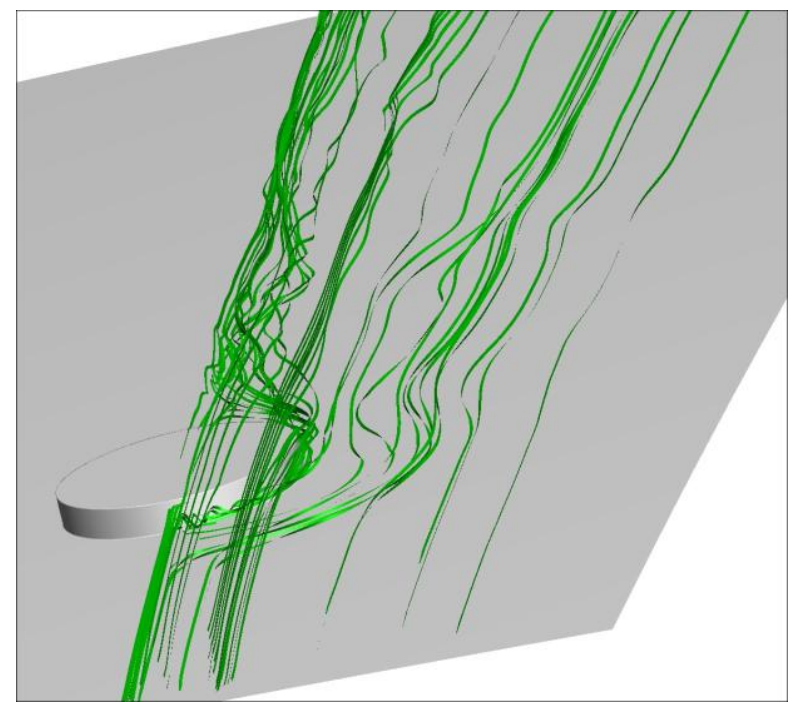

(a)

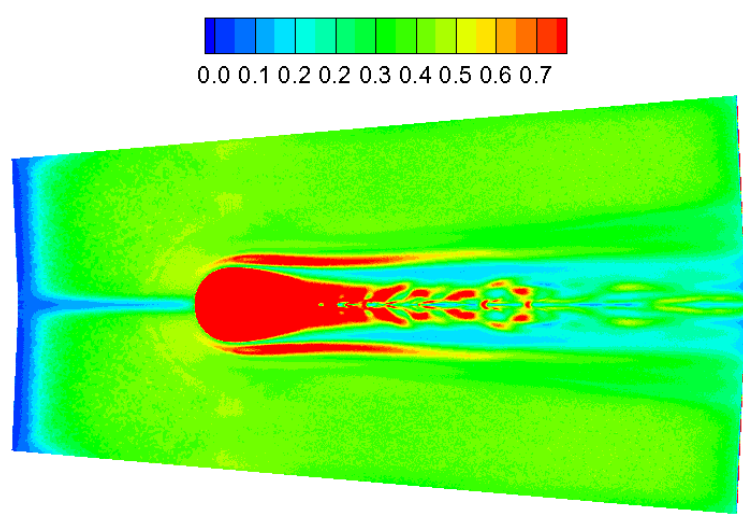

(b)

Figure 13. Instantaneous flow characteristics for the protuberance case (a) streamline patterns (b) vorticity contours at a constant wall-normal distance plane (with a wall distance of 1.1 times the protuberance height) 


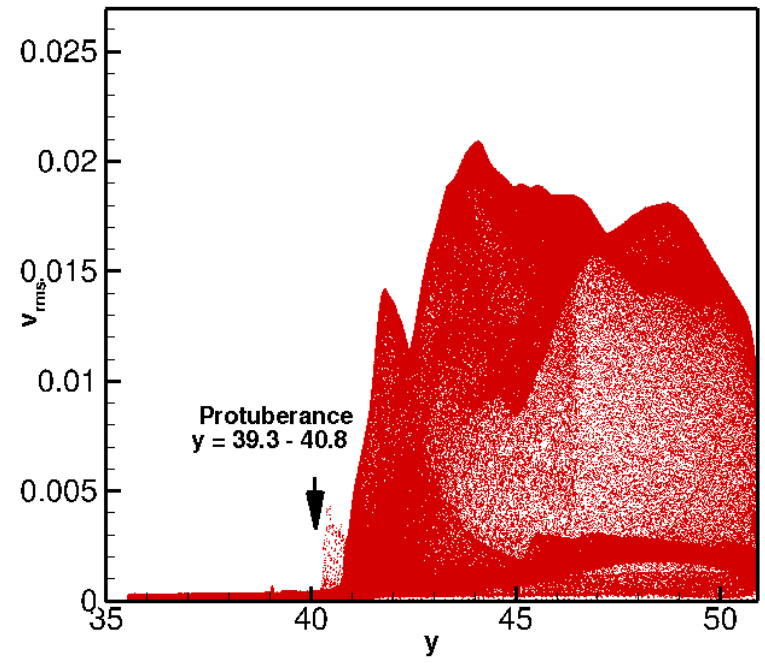

(a)

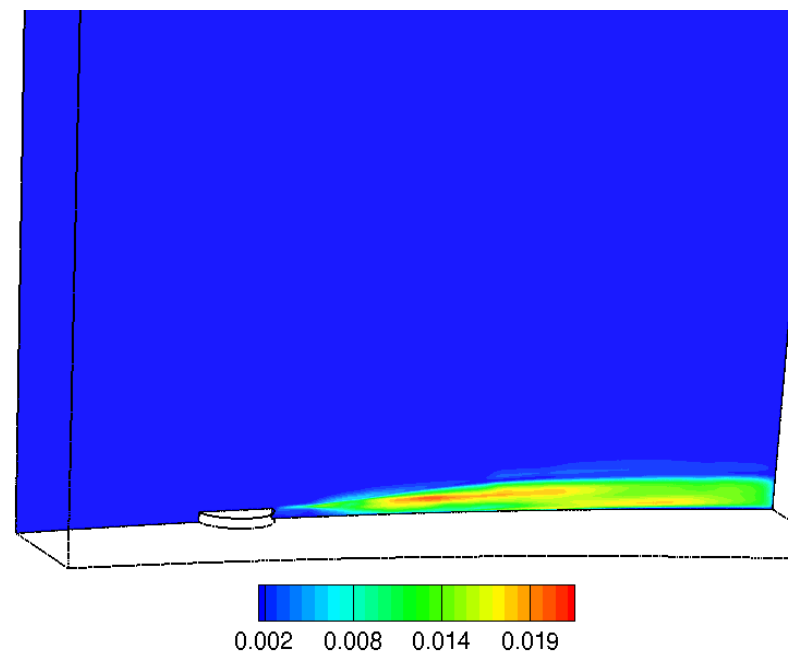

(b)

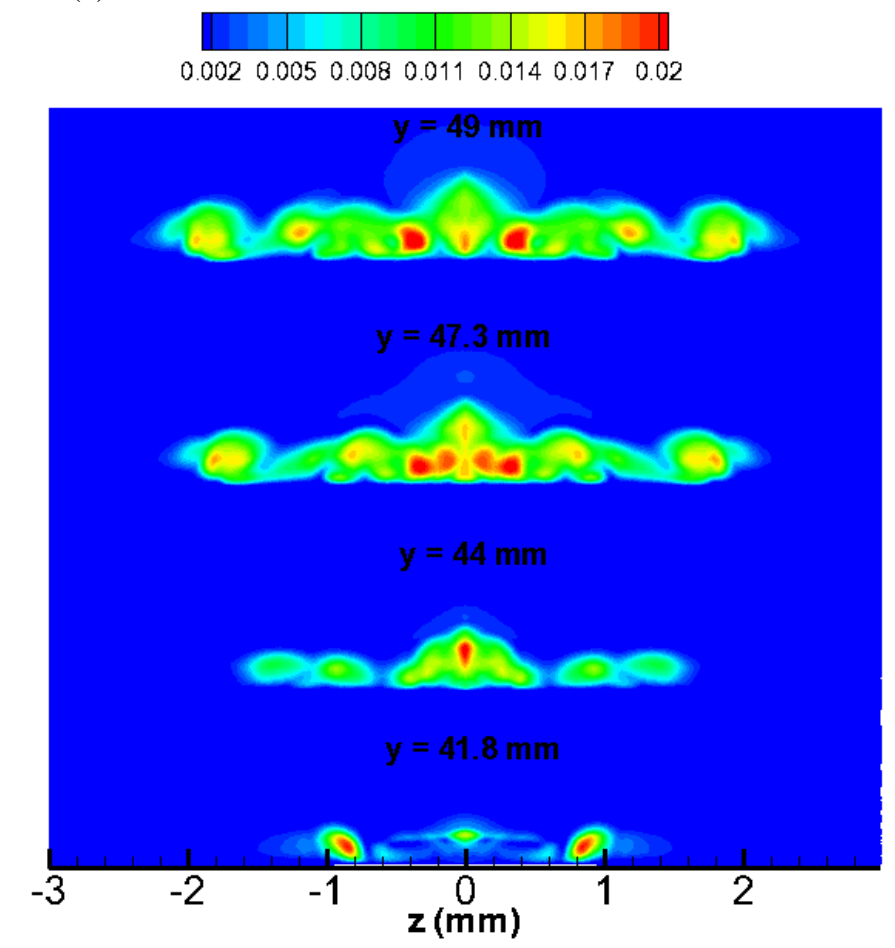

(c)

Figure 14. Computed rms v-velocity distribution for the protuberance case (a) $v_{\text {rms }}$ versus $y$-coordinate for all mesh points at the symmetry plane (b) $v_{\text {rms }}$ contours at the symmetry plane (c) $v_{\text {rms }}$ contours at several downstream constant y planes

To assess the effect of Reynolds number, preliminary computations were performed at a unit Reynolds number of $2.62 \times 10^{8} / \mathrm{m}$. By similarity scaling, the boundary layer thickness would be reduced to about $1 / \sqrt{10}$ of the original value and the mesh has to be refined accordingly. To avoid a significant increase in grid count and decrease in time steps, the inflow boundary layer thickness was kept the same as in the $R e_{k k}=800 \mathrm{case}$. This is equivalent to moving the protuberance or cavity to a downstream location where the boundary layer attains the specified thickness at the higher unit Reynolds number. As mentioned in the axisymmetric calculations, the resulting $k / \delta$ value is around 0.9 and the $R e_{k k}$ value also increases by roughly 10 times. Cavity results shown here have reached a quasi-steady 
periodic state, while those for the protuberance case have not. Nevertheless, the trend of increasing Reynolds number can be studied effectively for both cases. Figure 15 shows the computed vortical structure and surface heating distribution for the protuberance case. Comparison with Fig. 10(b) indicates that surface heating along the centerline is higher. High surface heating also sustains much further downstream. Multiple hairpin vortices appear downstream of the protuberance. These vortices break down into smaller and more refined structures. It is interesting to note that the extent of azimuthal spreading remains about the same. The vorticity intensity also appears to decrease as the shed vortices travel beyond about three diameters downstream in the wake region. It remains to be seen whether this decaying trend persists in the final asymptotic state. Flow characteristics for the cavity case computed at the higher Reynolds number are shown in Fig. 16. Unlike the $R e_{k k}=800$ case, spontaneous vortex shedding takes place when the Reynolds number is increased. As shown in Fig. 16(a), hairpin vortices similar to those shown in Fig. 12(a) are also present for the cavity case at a higher Reynolds number. Unlike the protuberance though, these hairpin vortices are narrower and more confined near the symmetry plane. Similar to the 2D cavity configurations, vortex shedding takes place right on top of the cavity, indicating that a shear-layer mode is responsible for the instability. Discrete high surface heating regions as shown in Fig. 16(b) are present for the cavity case. Vorticity contours in the symmetry plane (see Fig. 16(c)) clearly show the origin of the instability on top of the cavity. However, no regular high-shear pattern as observed in Fig. 11(a) exists for the cavity case. Time traces also reveal that disturbances for the cavity wake possess several spectral peaks, with the dominant one at around $68 \mathrm{kHz}$ (higher than the computed frequency for the 2D cavity). Results shown here indicate that the cavity at a higher Reynolds number would result in spontaneous vortex shedding and form hairpin vortices downstream. 

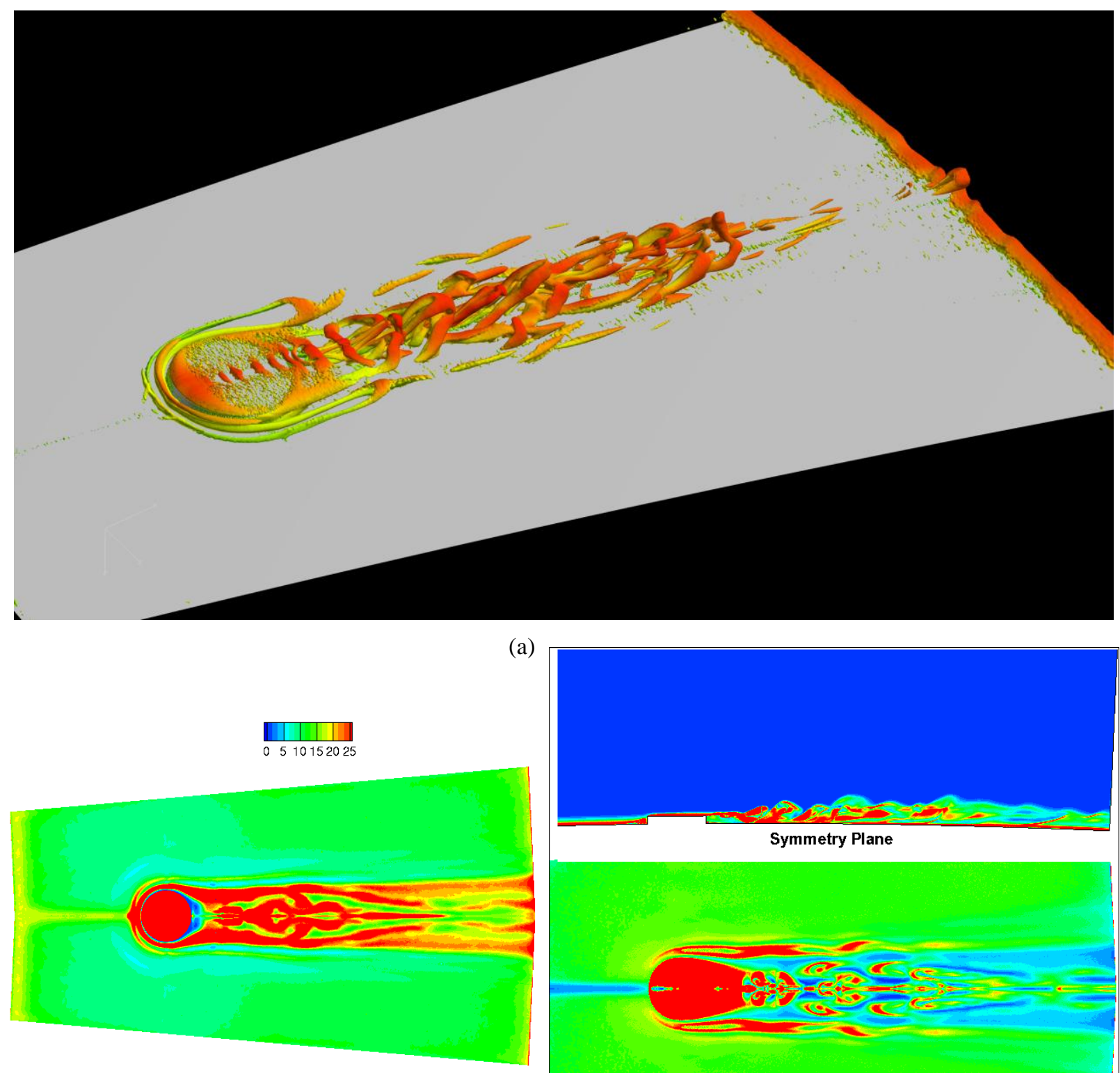

(b)

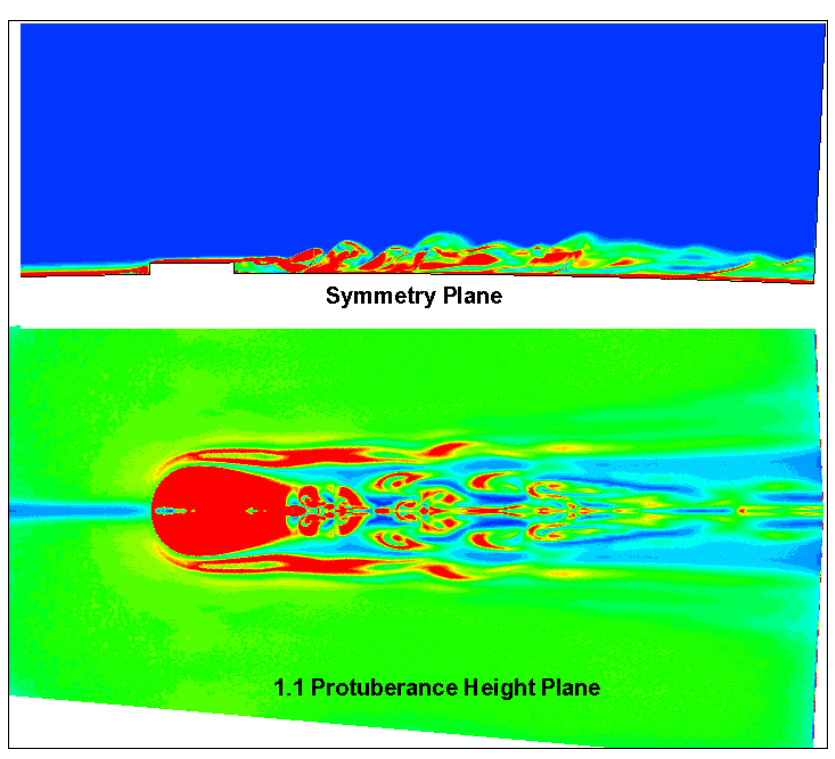

(c)

Figure 15. Flow characteristics for the protuberance computed at a unit Reynolds number of $2.62 \times 10^{8} / \mathrm{m}$ : (a) $Q$ value (non-dimensional value of 0.01 ) isosurfaces (b) surface normal temperature gradient (surface heat flux) (c) vorticity contours in the symmetry plane and a plane with a constant wall-normal distance of 1.1 protuberance height 


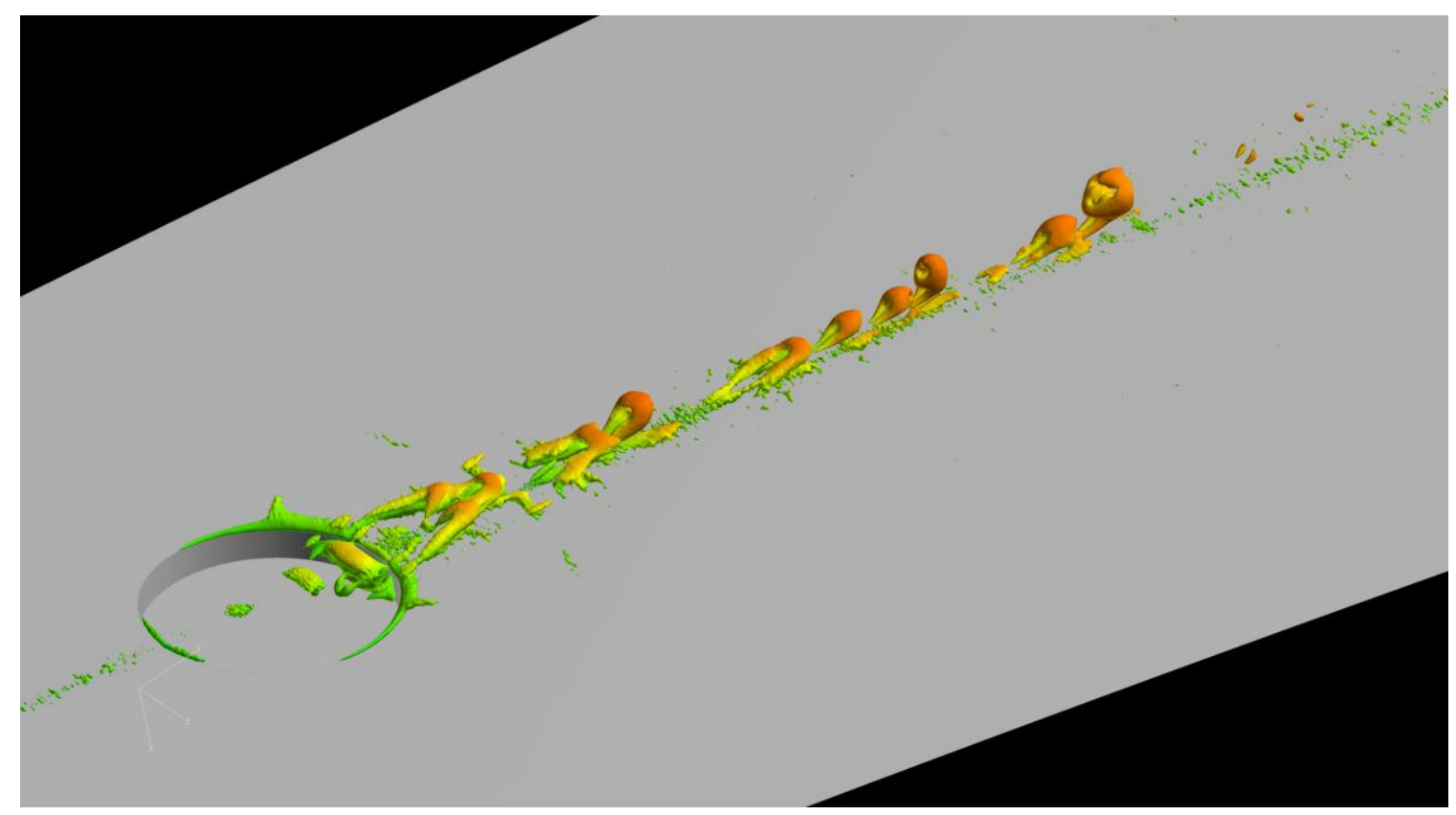

(a)

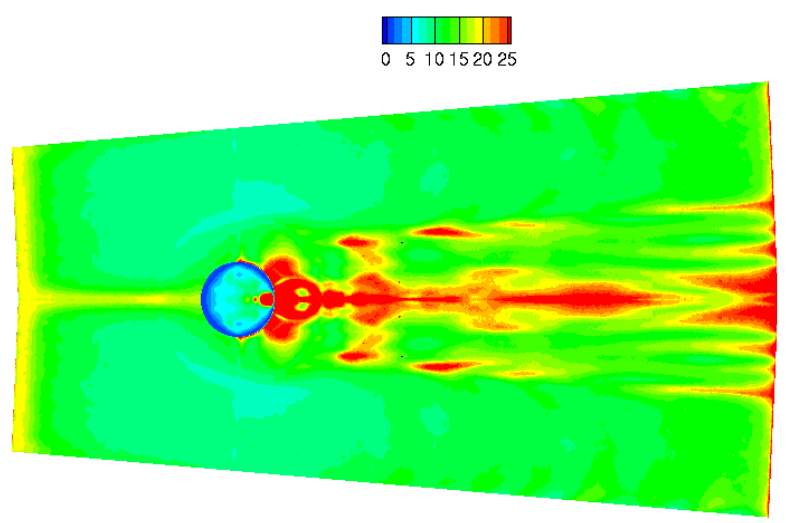

(b)

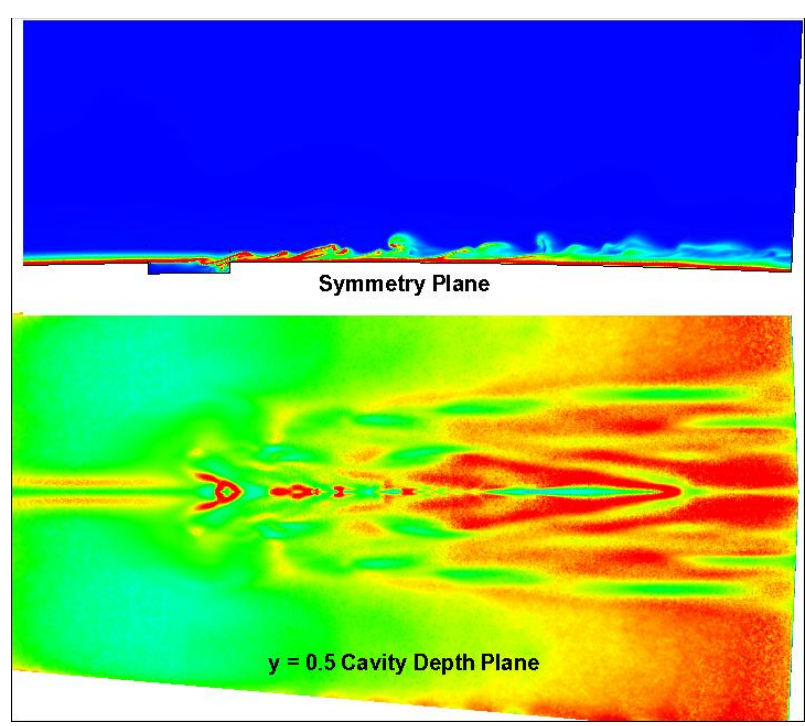

(c)

Figure 16. Flow characteristics of the cavity computed at a unit Reynolds number of $2.62 \times 10^{8} / \mathrm{m}$ : (a) $Q$ magnitude isosurfaces at a non-dimensional value of 0.01 (b) vorticity surface normal temperature gradient (c) vorticity contours at the symmetry and $y=0.5 k$ plane

\section{Purdue Mach 6 Nozzle Cylindrical Roughness Element}

The on-going series of NASA Langley Research Center experiments by Danehy and colleagues ${ }^{16}$ have been performed for various roughness sizes and shapes at a post-shock Mach number around 4-5. Planar laser induced fluorescence (PLIF) flow visualizations in these experiments provide detailed flow structures and evidence of possible laminar-turbulent transition in the wake region. In the recent Purdue University/Boeing/AFOSR Mach 6 Quiet Tunnel experiment, ${ }^{7}$ a slender cylindrical roughness element with a height to diameter ratio of about 5:3 (or $k / D=1.67)$ is placed in the laminar tunnel-wall boundary layer. In addition to surface flow visualization using temperature sensitive paint, hot-wire probes and Pitot tube measurements downstream of the wake are also available for quantitative comparisons with computations. Unsteady data at several probe locations downstream of the 
roughness element exhibits distinct disturbance peaks suggesting the possible presence of roughness wake instability waves.

A cylindrical roughness element with a diameter, $\mathrm{D}$, of $6 \mathrm{~mm}$ and a height of $10 \mathrm{~mm}$ is placed on the nozzle wall of the Purdue Mach 6 Quiet Tunnel at the location indicated by the green arrow in Fig. 17. At the location of the roughness element, the nozzle flow has approached an almost constant Mach number of about 5.91. Since the roughness size is very small compared to the nozzle radius of curvature, the flow around it can be modeled by a flat plate with a free stream Mach number of 5.91 with reasonable accuracy. To match the nozzle Reynolds number, a similarity boundary layer profile with the same boundary layer thickness as shown in Fig. 17(c) is used to simulate the flow conditions at the roughness location. The resulting roughness height to the boundary layer thickness is about 1.1. Therefore, a numerical computation is performed for a cylindrical roughness element on a flat plate with a free-stream Mach number of 5.91 and a temperature of $54 \mathrm{~K}$ (Fig. 17(a)). The wall temperature is set to be at 337 $\mathrm{K}$ and stagnation pressure is $90 \mathrm{psi}$. The inflow and outflow boundaries of the domain are located at $94 \mathrm{~mm}$ upstream and $154 \mathrm{~mm}$ downstream of the center of the cylinder, respectively. It should be noted that the approximated inflow profiles from flat-plate boundary-layer solutions and the flat-plate surface (as opposed to the curved, axisymmetric boundary layer along the nozzle wall) would result in differences in the predicted instability wave frequencies due to the differences in boundary layer thickness and similarity scales. Both settings would be dominated by the second-mode instability but the predicted disturbance frequency range will be different. The investigation of this "simulated" configuration in this paper is not to reproduce or verify the experimental data, but rather, to study fundamental transition physics in a similar setting.

The free-stream boundary in the wall-normal direction increases downstream to cope with the boundary-layer growth. For the present instability wave simulations, only half of the cylinder is computed with a spanwise boundary located at $20 \mathrm{~mm}$ away from the center. This computational domain rules out possible asymmetric instability modes. However, flow visualization from the experiments ${ }^{7}$ did not show evidence of such asymmetric modes. Computations were performed for several meshes. Results presented here were obtained with the finest mesh, which has about 54 million tetrahedrons (see Fig. 18(b) for a close-up view near the roughness element).

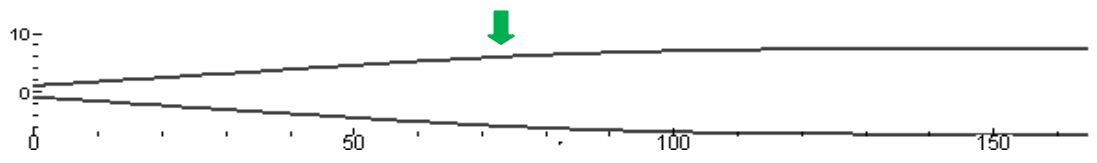

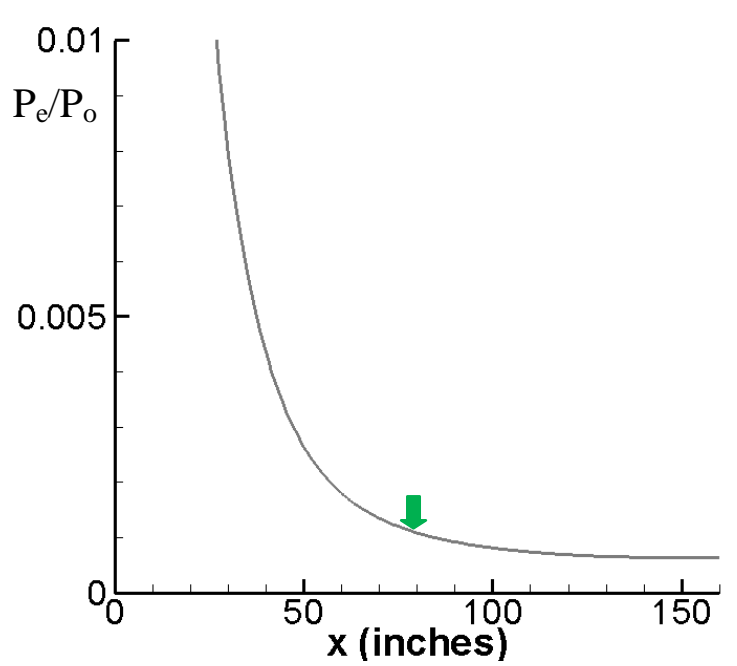

(b)

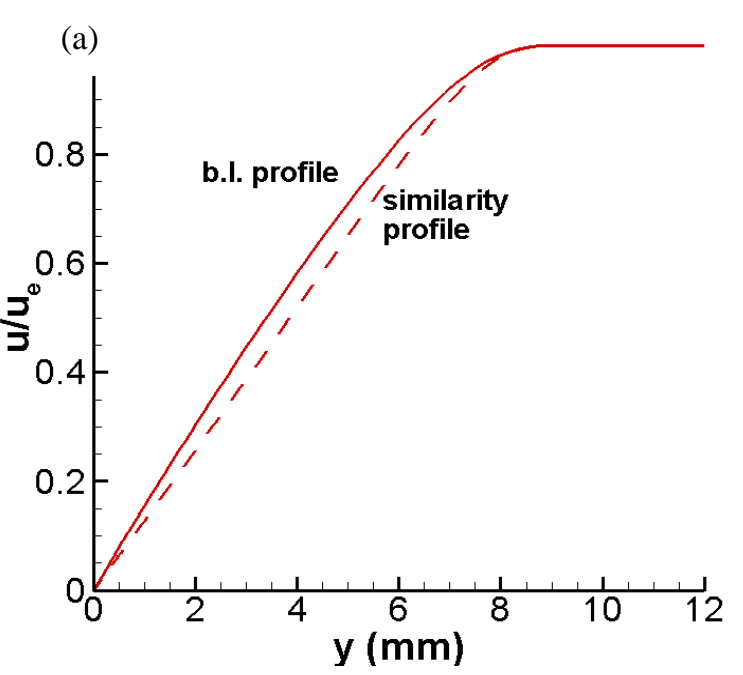

(c)

Figure 17. Nozzle wall and pressure distribution for the Purdue Boeing/AFOSR Mach 6 Quiet Tunnel (a) Nozzle wall and the roughness location (green arrow) (b) wall pressure distribution (c) matched similarity and boundary-layer profile at the roughness location 


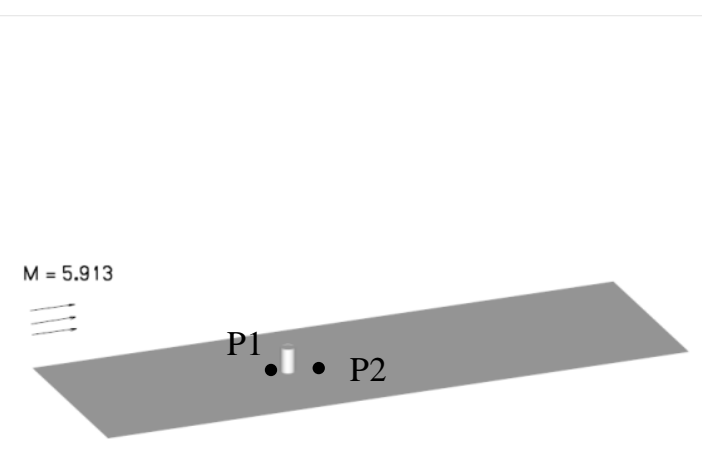

(a)

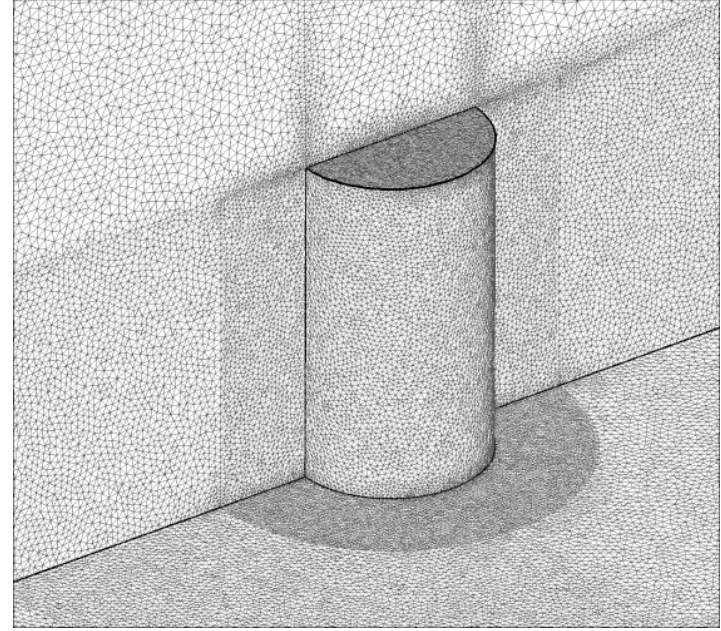

(b)

Figure 18. Computational domain for the cylindrical roughness element and the flat plate (a) domain (b) close-up view for the near cylinder surface mesh

To speed up the computations, local time-stepping was first run until numerical iterations settled to a semiconverged solution indicated by visually unchanged free-stream shock position and boundary layer thickness ahead of the roughness element. Time accurate computations were then turned on until a steady or quasi-steady state had been reached. The time history during iterations was monitored at several probe locations. Figure 19 shows the time history of pressure and its power spectra at two probe locations; Probe P1 is located at about 1D upstream of the roughness element and P2 is located about $1.75 \mathrm{D}$ downstream and $2.5 \mathrm{D}$ away from the spanwise symmetry plane (see Fig. 18(a)). Evidently, time-accurate solutions have reached an oscillatory quasi-steady state at both probe locations. For the current configuration with a roughness height to boundary layer thickness of 1.1, strong flow unsteadiness is still present, similar to that observed at a larger roughness height and smaller boundary layer edge Mach number (see Ref. 5). The power spectra reveal several peaks upstream of the roughness element. The dominant peak occurs at about $46 \mathrm{kHz}$ at both probe locations. This frequency is also present throughout the wake region. This frequency is higher than the $21 \mathrm{kHz}$ spectra peak observed in the experiments. ${ }^{7}$ The discrepancy can be attributed to the difference in meanflow profiles and flat-plate approximation used in the present computations. As mentioned above, the goal is not to match the measured frequency but to demonstrate that there exists a dominant tone in the wake region and to further explore the flow physics related to this instability wave.

Vortex structures ahead and behind the roughness element can be seen in Fig. 20 where the vorticity contours are plotted at several planes. Strong vortices are formed right upstream and downstream of the cylinder. In addition to the horse-shoe vortices wrapping around the cylinder, a strong vortex streak is formed right behind the cylinder. Streamwise development of the vortex pattern can be better visualized in Fig. 21(a). The spiral streamline patterns shown in Fig. 21(b) confirm the formation of strong vortices upstream of the cylinder. Figure 22 depicts vorticity contours at three wall-normal distances. The cylinder height is about $10 \mathrm{~mm}$. Therefore, these plots correspond to either close to the flat-plate surface or about half of the cylinder height. The horseshoe vortices become very unstable both upstream and downstream of the cylinder. Small structures begin to appear near the end of the domain. It is interesting to note that the streak right behind the cylinder appears to be more unstable (i.e., larger oscillatory amplitudes) than the horseshoe vortices. This is in contrast to what was observed in Ref.5 in which the horseshoe vortices became unstable earlier than the mushroom streak. 


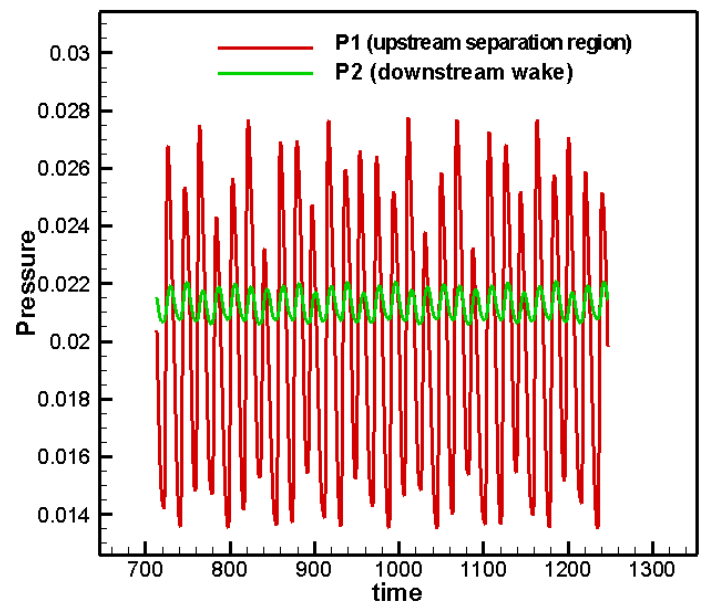

(a)

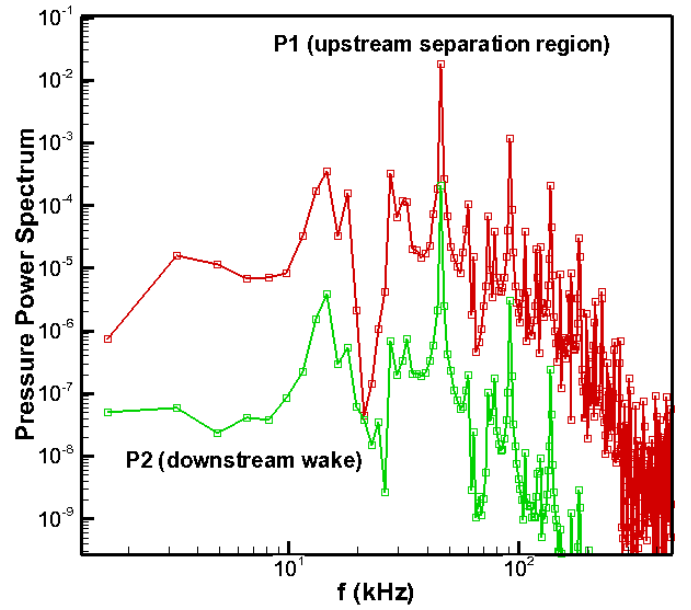

(b)

Figure 19. Time histories at two probe locations located upstream and downstream of the cylindrical roughness element: (a) pressure vs. non-dimensional time (b) pressure power spectra

The computed unsteady wake structure consists of one mushroom-like streak and a pair of crossflow-like horseshoe vortices. These vortices oscillate at a dominant frequency according to the time histories as shown in Fig. 19. The origin of this frequency appears to be the strong and localized instability taking place in the front side separation region of the cylinder as indicated by the spike around $x=-5$ (roughness element is located at $x=0$ ) in the streamwise rms u-velocity distribution shown in Fig. 23(a). This mechanism is quite similar to that observed for a larger and lower aspect ratio cylindrical roughness element, ${ }^{5}$ except that the height to the boundary layer thickness is slightly smaller in the present case. The disturbance field for a protuberance in a supersonic boundary layer shown here is distinctly different from that for the subsonic case shown in Fig. 14(a). In addition to the absolute instability like disturbance generation upstream of the protuberance, the disturbance field appears to undergo a second spurt downstream of the cylinder (but with smaller peaks) and then decay. Further downstream, the disturbances continue to grow again until the end of the domain. This final growth causes the perturbations to spread towards the wall as shown in Fig. 23(b), indicating the flow is evolving towards a transitional state.

Figure 24 shows the $\mathrm{Q}$ value isosurfaces at a non-dimensional value of 0.001 around the cylindrical protuberance. Both the horseshoe vortices and the wake streak remain intact for some distance downstream of the cylinder due to the supersonic expansion around the cylinder. Further downstream, the flow instability causes the streak to break down into a smaller ring-like structure. The consecutive rings wrapping around the main streak in the wake region mark the distinct feature of the wake instability of supersonic boundary layer. Two main horseshoe vortices also become twisted downstream of the cylinder. Both the streak and horseshoe vortices break down into much smaller structures near the end of the domain. Similar structures were also observed in the previous computations ${ }^{5}$ for NASA LaRC's PLIF experiments. ${ }^{16}$ In contrast to the subsonic cases shown previously, the vortical structure upstream of the roughness is also distinctively different. Multiple vortices co-exist upstream and they are responsible for disturbance generation and frequency selection. ${ }^{5}$ These differences in subsonic and supersonic mechanisms may lead to different path of transitional and turbulent flow propagation downstream. Generally speaking, the minimal protuberance height to the boundary layer thickness ratio required to trigger transition is higher for supersonic boundary layers. Simulations were also performed by converting the slender roughness into a cylindrical cavity under the same free-stream conditions. No flow instability was found for this small diameter cavity. A shallower and wider cavity is expected to be more unstable, ${ }^{26}$ which will be a topic for future research. 


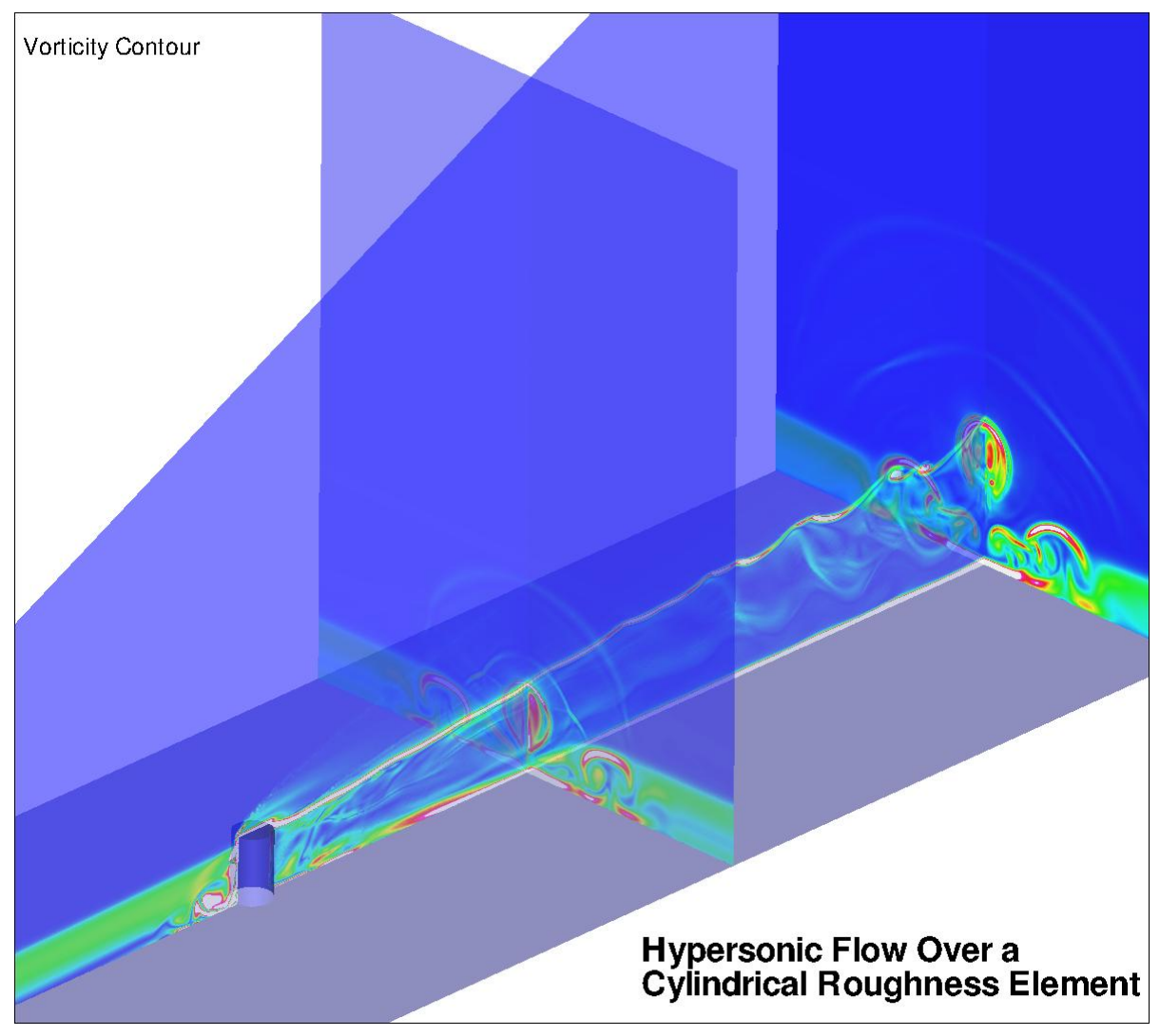

Figure 20. Vorticy contours at several planes for the Mach 6 cylindrical roughness element 


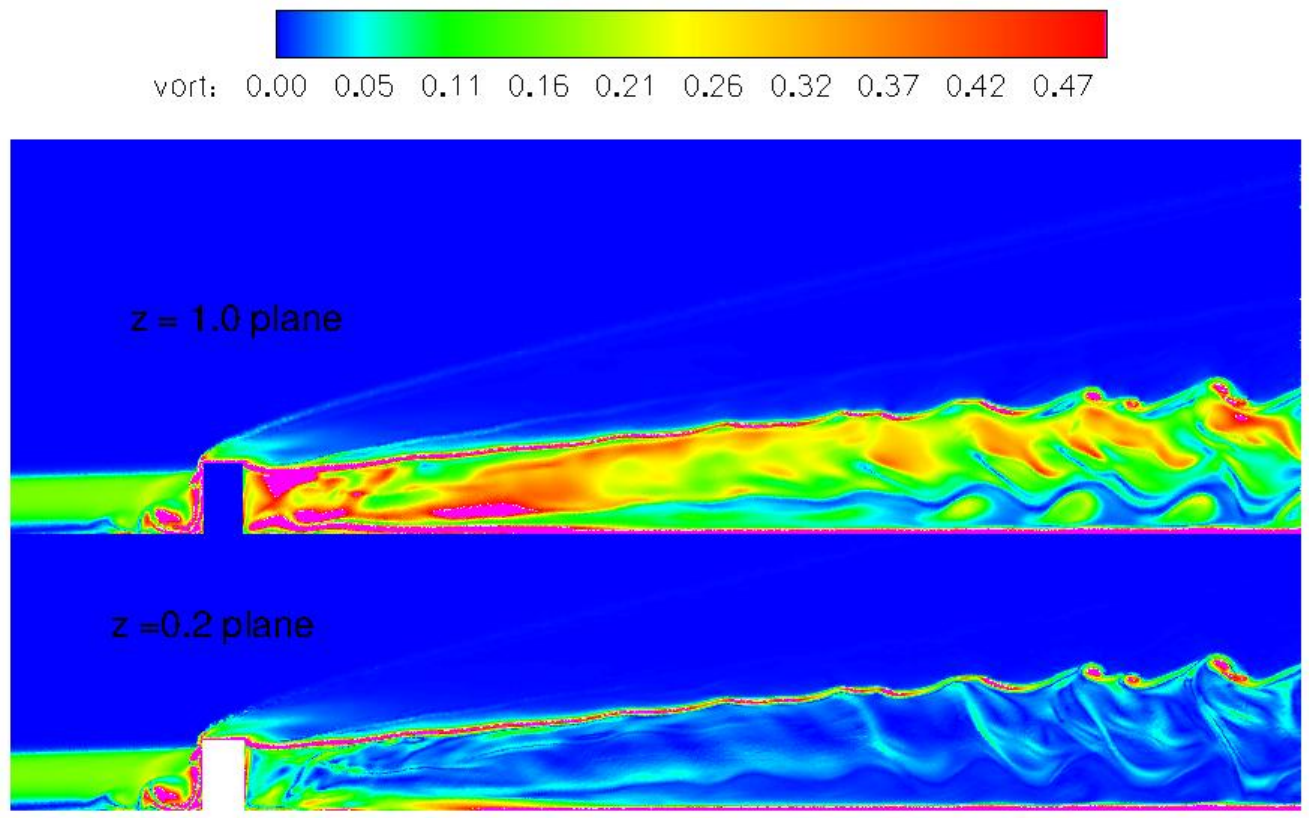

(a)

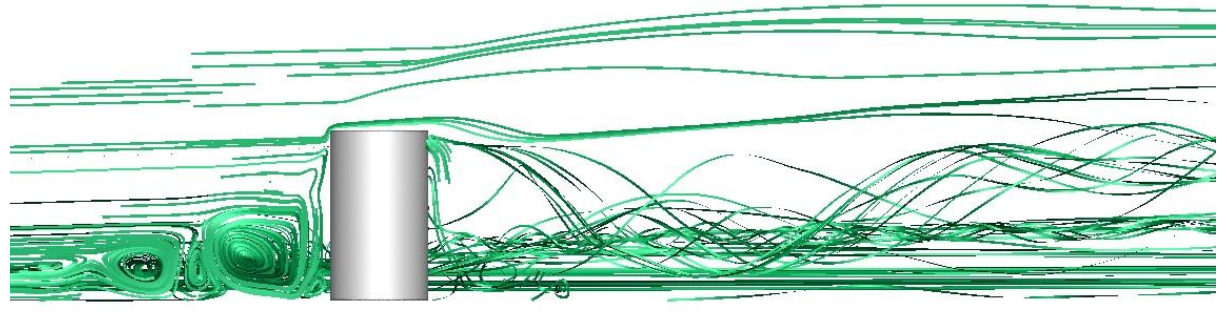

(b)

Figure 21. Vortical structures near the symmetry plane, showing (a) vorticity contours at the symmetry plane (b) streamline patterns near the roughness element

American Institute of Aeronautics and Astronautics 
(a)

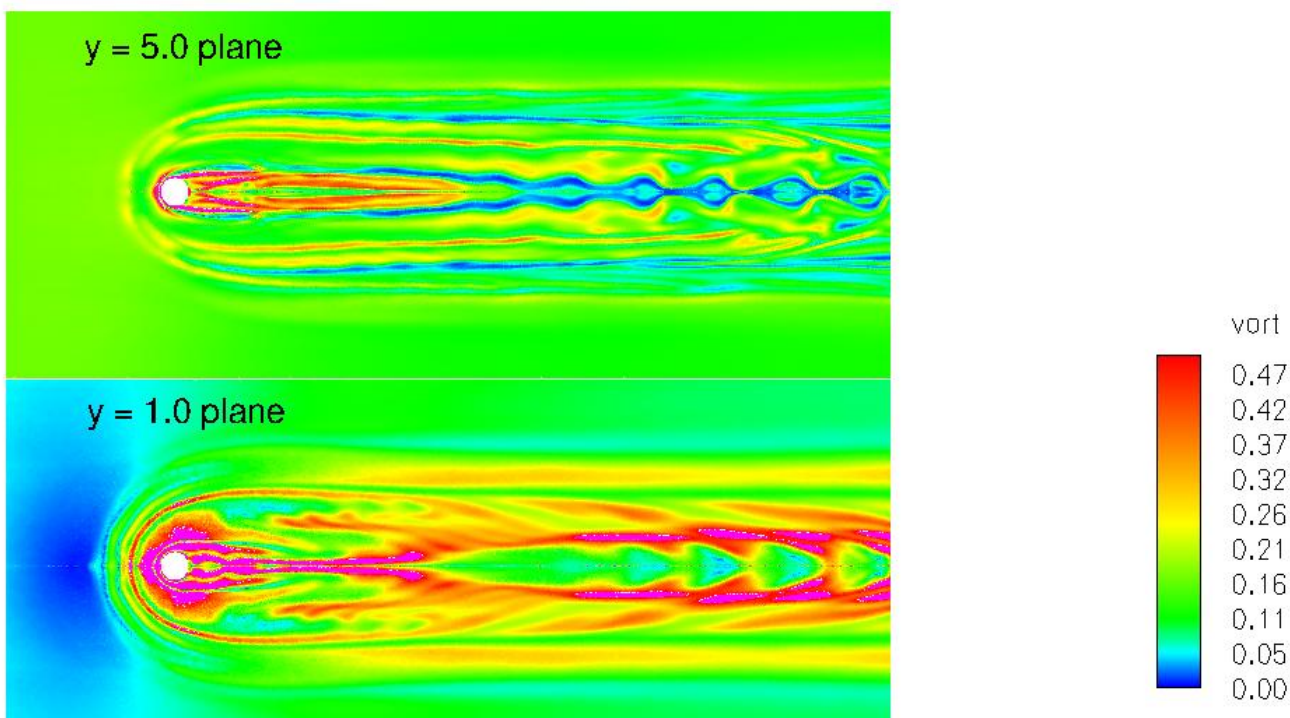

(c)

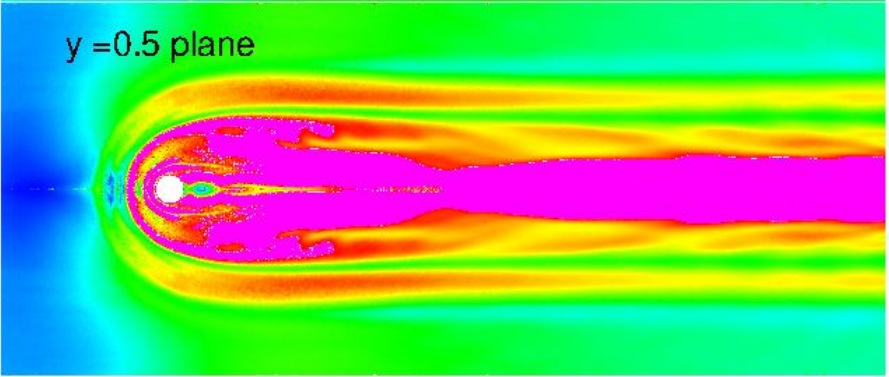

Figure 22. Vorticity contours at several horizontal planes (a) y $=5 \mathrm{~mm}$ (b) y = 1.0 $\mathrm{mm}(\mathrm{c}) \mathrm{y}=0.5 \mathrm{~mm}$

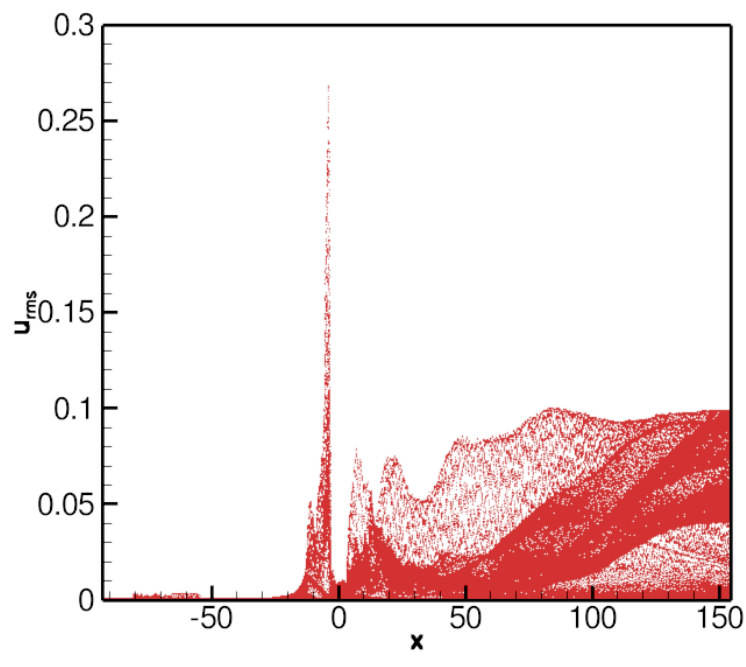

(a)

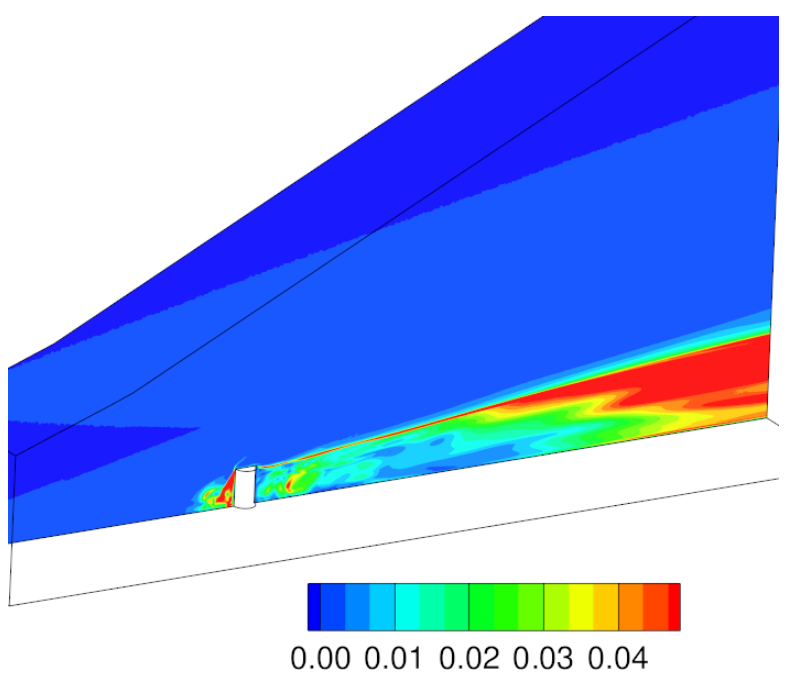

(b)

Figure 23. Computed rms v-velocity distribution for the Mach 6 cylindrical roughness element (a) $\mathbf{v}_{\text {rms }}$ versus $x$-coordinate for all mesh points at the symmetry plane $(b) v_{r m s}$ contours at the symmetry plane 


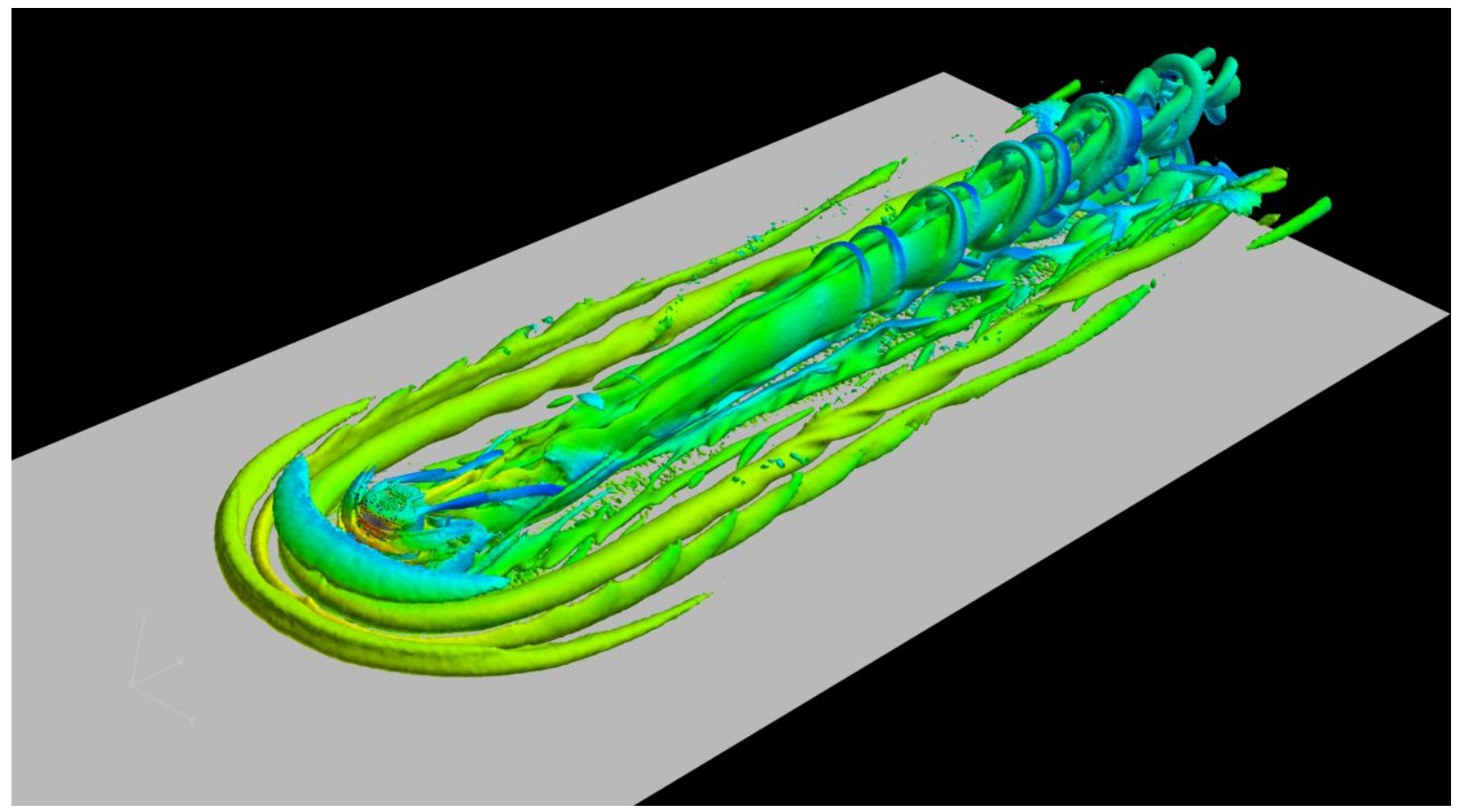

Figure 24. Q (second invariant of the velocity gradient tensor) isosurfaces color shaded by the temperature for the Mach 6 cylindrical protuberance, showing vortical structure development downstream of the slender roughness element

\section{Summary}

Effects of surface irregularities in the form of a protuberance and cavity on hypersonic vehicle transition are investigated numerically using the unstructured-mesh, space-time CESE method. Due to the large bluntness in a CEV-like vehicle, these surface irregularities reside in an accelerating subsonic boundary layer. A computational domain involving supersonic free-stream and subsonic inflow and outflow was used to simulate a Mach 6 flow over the CEV wind-tunnel model with an isolated protuberance or cavity embedded in the subsonic region with a boundary-layer edge Mach number of about 0.3 . It has been demonstrated that numerical computations for a portion of the vehicle surface including the irregularities were able to hold the bow shock in the correct position in order to simulate the same flow conditions for the full vehicle. Such truncated domain allows more efficient time-accurate simulations of small-scale features around the surface irregularities. For the Mach 6 wind tunnel boundary conditions with a roughness Reynolds number $R e_{k k}$ of 800 , it was found that a protuberance with a height to boundary layer thickness ratio of 0.73 leads to strong wake instability and spontaneous vortex shedding while a cavity with identical geometry causes only mild flow unsteadiness. The same cavity with a larger Reynolds number also leads to similar spontaneous vortex shedding and wake instability. The wake development and the formation of hairpin vortices were found to be qualitatively similar to that observed for an isolated hemisphere submerged in a subsonic, low speed flat-plate boundary layer. However, the shed vortices and their accompanying instability waves were found to be slightly stabilized downstream by the accelerating boundary layer along the CEV surface. Similarities and differences in the wake instability of a protuberance and a cavity are investigated. In addition, the study of supersonic flow over a slender cylindrical roughness element exemplifies a very different flow regime that is often encountered for slender shaped hypersonic vehicles. It has been shown that the wake flow structure behind a roughness element at supersonic edge Mach number is distinctly different from its subsonic counterpart. Results from high-fidelity simulations of both types of instability mechanisms can be used to enhance our understanding of the fundamental physics, which in turn, would improve the predictive capability. 


\section{Acknowledgments}

The authors thank Dr. Deepak Bose of the NASA Ames Research Center and Dr. Sin-Chung Chang of the NASA Glenn Research Center for technical discussions and Andrew Norris of NASA Langley Research Center for assistance in grid generation. The research funding for Balaji Venkatachari is provided by the Hypersonic Project under the Fundamental Aeronautics Program (FAP). Three NASA authors acknowledge the NASA FTEs provided by the AAAP discipline of the Hypersonics project.

\section{References}

${ }^{1}$ Duan, L., Wang, X., and Zhong, X., "A High-Order Cut-Cell Method for Numerical Simulation of Hypersonic-Boundary Transition with Surface Roughness," AIAA Paper 2008-3732, 2008.

${ }^{2}$ Marxen, O. and Iaccarino, G., "Simulation of the Effect of a Roughness Element on High-speed Boundarylayer Instability," AIAA Paper 2008-4400, 2008.

${ }^{3}$ Chang, C.-L. and Choudhari, M. M., "Hypersonic Viscous Flow over Large Roughness Elements," AIAA Paper 2009-0173, 2009, Theo. Comput. Fluid Dynamics, Vol. 25, Issue 1, pp. 85-104, 2011.

${ }^{4}$ Choudhari, M., Li, F., and Edwards, J.E., "Stability Analysis of Roughness Array Wake in a High-Speed Boundary Layer," AIAA Paper 2009-0170, 2009.

${ }^{5}$ Chang, C.-L., Choudhari, M. M., and Li, F., "Numerical Computations of Hypersonic Boundary-Layer over Surface Irregularities," AIAA Paper 2010-1572, 2010.

${ }^{6}$ Bartkowicz, M. D., Subbareddy, P. K., and Candler, G. V., "Numerical Simulations of Roughness Induced Instability in the Purdue Mach 6 Wind Tunnel," AIAA Paper 2010-4723, 2010.

${ }^{7}$ Wheaton, B. M. and Schneider, S. P., "Roughness-Induced Instability in a Laminar Boundary Layer at Mach 6," AIAA Paper 2010-1574, 2010.

${ }^{8}$ Berry, S. A., Horvath, T. J., Greene, F. A., Kinder, G. R., and Wang, K. C., "Overview of Boundary Layer Transition Research in Support of Orbiter Return to Flight," AIAA Paper 2006-2918, 2006.

${ }^{9}$ Horvath, T. J., Berry, S. A., Merski, N. R., Berger, K. T., Buck, G. M., Leichty, D. S., and Schneider, S. P., "Shuttle Damage/Repair from the Perspective of Hypersonic Boundary Layer Transition - Experimental Results," AIAA Paper 2006-2919, 2006.

${ }^{10}$ Chang, S.-C., "The Method of Space-Time Conservation Element and Solution Element-A New Approach for Solving the Navier-Stokes and Euler Equations," J. Comput. Physics, Vol. 119, pp.295-324, 1995.

${ }^{11}$ Chang, S.-C. and Wang, X.-Y., "Multi-Dimensional Courant Number Insensitive CE/SE Euler Solvers for Applications Involving Highly Non-uniform Meshes," AIAA Paper 2003-5285, 2003.

${ }^{12}$ Chang, C.-L., "Time-Accurate, Unstructured-Mesh Navier-Stokes Computations with the Space-Time CESE Method," AIAA Paper 2006-4780, 2006.

${ }^{13}$ Chang, C.-L., "Three-Dimensional Navier-Stokes Calculations Using the Modified Space-Time CESE Method," AIAA Paper 2007-5818, 2007.

${ }^{14}$ Rowley, C.W, Colonius, T., and Basu, A.J., "On Self-Sustained Oscillations in Two-Dimensional Compressible Flow over Rectangular Cavities," Journal of Fluid Mechanics, Vol. 455, pp. 315-346, 2002.

${ }^{15}$ Krishnamurty, K., "Sound Radiation from Surface Cutouts in High Speed Flow," PhD thesis, California Institute of Technology, 1956.

${ }^{16}$ Danehy, P.M., Garcia, A.P., Borg, S., Dyakonov, A.A., Berry, S.A., Inman, J.A., and Alderfer, D.W., "Fluorescence Visualization of Hypersonic Flow Past Triangular and Rectangular Boundary-Layer Trips," AIAA Paper 2007-536, 2007.

${ }^{17}$ Amar, A. J., Horvath, T. J., Hollis, B. R., Berger, K. T., Berry, S. A., and Calvert, N., "Protuberance Boundary Layer Transition for Project Orion Crew Entry Vehicle," AIAA Paper-2008-1227, 2008.

${ }^{18} \mathrm{http}: / /$ vulcan-cfd.larc.nasa.gov/index.html (November 15, 2010)

${ }^{19}$ Reda, D. C., Wilder, M. C., and Prabhu, D. K., "Transition Experiments on Blunt Bodies with Isolated Roughness Elements in Hypersonic Flight," J. Spacecraft and Rockets, Vol. 47, No. 5, pp. 828-835, 2010.

${ }^{20}$ Roos, F. W. and Kegelman, J. T., "Control of Coherent Structures in Reattaching Laminar and Turbulent Shear Layers," AIAA J. Vol. 24, No. 12, pp. 1956-1963, 1986.

${ }^{21}$ Tufo, H. M, Fischer, P. F., Papka, M. E., and Blom, K., "Numerical Simulation and Immersive Visualization of Hairpin Vortices," in Proc. of the ACM/IEEE SC99 Conf. on High Performance Networking and Computing, IEEE Computer Soc., CDROM (1999).

${ }^{22}$ Choudhari, M. and Fischer,P., "Roughness-Induced Transient Growth,” AIAA Paper 2005-4765, 2005. 
${ }^{23}$ Acarlar, M.S. and Smith, C.R., "A Study of Hairpin Vortices in a Laminar Boundary Layer. Part 1," J. Fluid Mech., Vol. 175, pp. 1-42, 1987.

${ }^{24}$ Klebanoff, P., Cleveland, W.G., and Tidstrom, K.D., "On the Evolution of a Turbulent Boundary Layer Induced by a Three-Dimensional Roughness Element," J. Fluid Mech., Vol. 237, pp. 101-187, 1992.

${ }^{25}$ Kosorygin, V. S., Crouch, J. D., and Ng, L.L., "Transition movement in the wake of protruding and recessed three-dimensional surface irregularities," Proceedings of the Seventh IUTAM Symposium on LaminarTurbulent Transition, Stockholm, Sweden, 2009, Schlatter, P. and Henningson, D. S. (Eds.), Springer-Verlag, Berlin, 2010.

${ }^{26}$ Everhart, J. L., "Turbulent Supersonic/Hypersonic Heating Correlations for Open and Closed Cavities," J. Spacecraft and Rockets, Vol. 47, No. 4, pp. 545-553, 2010.

${ }^{27}$ Hunt, J. C. R., Wray, A. A., and Moin, P., "Eddies, stream, and convergence zones in turbulent flows," Center for Turbulence Research Report CTR-S88, 1988. 